\title{
High serum IgA and proportion of activated Th17 and Treg predict efficacy of abatacept in patients with early, seropositive rheumatoid arthritis
}

Jun Inamo

Keio University School of Medicine https://orcid.org/0000-0002-9927-7936

Yuko Kaneko ( $\nabla$ ykaneko.z6@keio.jp)

Keio University School of Medicine https://orcid.org/0000-0003-2646-5765

Jun Kikuchi

Keio University School of Medicine

Tsutomu Takeuchi

Keio University School of Medicine

Research article

Keywords: rheumatoid arthritis, abatacept, biomarker, precision medicine

Posted Date: March 9th, 2020

DOI: https://doi.org/10.21203/rs.3.rs-16418/v1

License: (c) (i) This work is licensed under a Creative Commons Attribution 4.0 International License. Read Full License

Version of Record: A version of this preprint was published at Clinical Rheumatology on March 11th, 2021. See the published version at https://doi.org/10.1007/s10067-021-05602-0. 


\section{Abstract}

Background To identify the predictive biomarkers for achieving remission with abatacept in patients with seropositive rheumatoid arthritis (RA).

Methods We enrolled patients with RA who were treated with abatacept. We compared the baseline laboratory results and longitudinal immune-phenotyping data between patients who achieved remission and those who did not achieve remission at 6 months according to the clinical disease activity index.

Results One hundred and twenty RA patients were enrolled. In the seropositive patients with early RA, high serum IgA levels, anti-CCP titers and neutrophil counts before treatment were predictors of remission (area under the curve [AUC], 0.659, 0.741, and 0.704, respectively). Additionally, activated Th17 (aTh17) cells and activated Treg (aTreg) cells before treatment were found to be significantly higher in patients with remission compared to those without remission $(2.9 \%$ vs $1.1 \%, P=0.02 ; 34.3 \%$ vs $17 \%, P=0.03$, respectively). The measurement of longitudinal cell subpopulation revealed a decrease in the effector CD4 T cell population after abatacept treatment, which correlated with anti-CCP titers and neutrophil counts, and was associated with remission achievement. In seropositive patients with established RA, high RF titers and low IFN-y levels may be possible biomarkers for remission.

Conclusion Our study has shown that serum IgA levels, anti-CCP titer and neutrophil counts are predictive biomarkers for evaluating the response to abatacept in patients with seropositive and early RA, and may reflect the inhibition of effector CD4 T cell subpopulations by abatacept.

\section{Background}

The advent of disease-modifying anti-rheumatic drugs has brought about a dramatic paradigm shift in the management of rheumatoid arthritis (RA). Currently, the goal for RA treatment is to achieve clinical remission, which has been facilitated by the development of various types of biological agents.

Abatacept is an effective biological agent with a unique mechanism of action on the immune cells [1]. For complete activation, T lymphocytes, the essential immune cells involved in the pathogenesis of RA [2], require co-stimulatory signals from antigen-presenting cells via the interaction of CD28 on T cells with CD80/86 on antigen-presenting cells. Abatacept is a soluble fusion protein consisting of an extracellular domain from the human cytotoxic T-lymphocyte-associated antigen 4 (CTLA4) and a modified $\mathrm{F}_{\mathrm{c}}$ portion from the human immunoglobulin (lg) G1 [1], which interferes with T cell activation by competing against CD28 via the CTLA4 domain [3]. Many trials have shown the efficacy of abatacept in RA, especially in patients with seropositivity and shorter disease duration [4-7]. However, some patients with seropositive RA do not respond to abatacept, which poses a challenge in the prediction of the effectiveness of abatacept treatment.

The aim of this study was to identify predictive biomarkers for the achievement of remission with abatacept in patients with seropositive RA. 


\section{Methods}

\section{Patients}

We enrolled patients diagnosed with RA based on the 2010 ACR/EULAR criteria who had undergone abatacept treatment from the year 2010 to the year 2018 in the Keio University Hospital [8]. Written informed consent was obtained from all the patients in accordance with the Declaration of Helsinki protocol. This study was approved by the ethics committee of our institution (the Ethics Committee of Keio University School of Medicine; 2009173).

\section{Data collection and definition}

The following laboratory parameters were collected from the patients' charts during initiation of the abatacept treatment and at 6 months post-treatment: tender joint count (TJC), swollen joint count (SJC), Health Assessment Questionnaire Disability Index (HAQ-DI), patient global assessment (PGA) using a visual analogue scale (VAS), evaluator global assessment using VAS (EGA), C-reactive protein (CRP), erythrocyte sedimentation rate (ESR), matrix metalloproteinase-3 (MMP-3), RF, IgG, IgA, $\lg$ M, counts of white blood cells (WBCs) and neutrophils, and the doses of methotrexate and prednisolone administered to the patients.

Seropositivity was defined according to the status of rheumatoid factor (RF) and anti-citrullinated peptide (CCP) as follows: seropositive RA, positive for RF and/or anti-CCP; seronegative RA, negative for both. Further, we divided seropositive patients into two groups according to the stage of RA; early RA was defined as a stage wherein patients, less than two years from the diagnosis of RA, presented with less than three erosions visible in the X-ray reports of the hands and feet of the patient; the patients were otherwise classified and defined as established RA [9]. Disease activity was calculated according to the clinical disease activity score (CDAl), and remission was defined as a CDAI $<2.8$ [10].

\section{Flow cytometry analysis}

Flow cytometry analysis was performed prospectively using samples from a part of patients at treatment initiation and 6 months post-treatment. Peripheral blood mononuclear cells were separated using density gradient centrifugation with the Ficoll-Paque Plus (GE Healthcare, Uppsala, Sweden) and cryopreserved in the CELLBANKER 1 (Nippon Zenyaku Kogyo, Fukushima, Japan). The antibodies used for staining peripheral blood mononuclear cells were as follows; anti-CD4-VioGreen (Miltenyi Biotec, Bergisch Gladbach, Germany); anti-CD3-Pacific Blue/fluorescein isothiocyanate (FITC), anti-CD8-Pacific Blue, antiCD14-(APC)-Cy7, anti-CD20 allophycocyanin-cyanine 7 (APC-Cy7), anti-CD25 phycoerythrin (PE)-Cy5, antiCD27-PE-Cy7, anti-CD38-PE-Cy5, anti-CD45RO-PE-Cy7, anti-CD56-PE/PE-Cy7, anti-CD80-FITC, anti-CD86PE-Cy5, anti-CD127-FITC, anti-CD161-APC and anti-chemokine (C-X-C motif) receptor 3 (CXCR3)-PE (all from BD Biosciences, Franklin Lakes, NJ, USA); anti-CD16-Brilliant Violet 510 and anti-CCR6-Brilliant Violet 421 (both from BioLegend, San Diego, CA, USA); and anti-mouse immunoglobulin G isotypematched controls (VioGreen from Miltenyi Biotec, the others from BD Biosciences). The peripheral cell subsets identified have been described in Supplementary table 1. 


\section{Statistical analysis}

We compared the variables between patients with remission and those without remission to screen for remission-related key features. The means of the continuous variables were compared using the Student's t-test, and the proportions were compared using the Fisher's exact test. Correlations were evaluated using the Spearman's correlation coefficients.

While analyzing the peripheral immuno-phenotyping and cytokines, we screened them with emphasis on the performance to divide the patients into subgroups based on whether remission was achieved or not using a combination of the Cox regression model-related feature selection and clustering analysis [11]. In detail, first, we applied feature dimension reduction to both the datasets based on the Cox regression model for obtaining the cumulative proportion of remission achievement and to remove irrelevant cell subpopulations as well as cytokines. This process enabled us to select remission-related features from multi-variate datasets. Second, we created subgroups of patients by consensus clustering, which is an unsupervised subtypes discovery method, using selected features from the immune-phenotyping dataset and the cytokine dataset, respectively [12]. Third, to aggregate the subgroups of patients according to each data set, we used similarity network fusion [13]. We checked how well the patients matched with their identified subgroup compared to other subgroups using a Silhouette width [14]. A high Silhouette value indicates that the sample is well-matched. Additionally, to visualize the relationships among selected features and subgroups of patients, we used biplot by applying the principal component analysis [15]. Thereby, we were able to efficiently generate subgroups of patients with heterogeneous characteristics.

To investigate the similarity between different types of biomarkers, we used multi-dimensional scaling (MDS) [16], a statistical technique involving non-linear dimensionality reduction, to reveal the relationships or patterns among large numbers of variables by reconstructing similarities between pairs of these variables in a reduced space.

$P<0.05$ was considered as statistically significant. All statistical analyses were performed using the $R$ software version 3.6.1 (R Foundation for Statistical Computing, Vienna, Austria).

\section{Results}

\section{Patient characteristics}

In this study, 120 RA patients were enrolled (17 seronegative RA, and 103 seropositive RA, Supplementary Table 2). The proportion of the achieved remission tended to be lower in the seronegative patients compared to that in the seropositive patients ( $17.6 \%$ vs $28.2 \%$, respectively) in spite of lower disease activity before treatment in the seronegative RA patients (disease activity for 28 joints, 3.79 vs $5.1, P<$ 0.01 ), indicating that patients with seronegative RA may not respond to abatacept treatment as compared to the response in patients with seropositive RA. 
To investigate the biomarkers associated with the efficacy of abatacept treatment other than seropositivity, we focused on the seropositive RA patients. Based on the definition stated in methods, patients were divided into two groups consisting of 24 early RA patients and 79 established RA patients (Supplementary Table 3). The patients in the early RA group responded better to abatacept treatment than those in the established group throughout 6 months of treatment despite high disease activity at the time of abatacept treatment initiation at the baseline with CDAl at 25.1 and CDAl at 18.3 in the early RA group and the established RA group, respectively (Figure 1B). The proportion of CDAI remission at 6 months post-treatment was higher in the early RA group than the CDAI proportion in the established RA group (37.5\% and $25.3 \%$, respectively), although the difference was not significant $(P=0.30)$.

\section{Predictive clinical biomarkers for evaluating response to abatacept}

We compared the baseline clinical characteristics and laboratory findings between patients who achieved CDAl remission at 6 months and those who did not achieve CDAl remission, separately in the early group and in the established group (Supplementary Table 4). In the early group, patients with remission tended $(P<0.15)$ to show higher serum IgA levels, anti-CCP titer, and counts of white blood cells and neutrophils than patients without remission $(351 \mathrm{mg} / \mathrm{dL}$ vs $289 \mathrm{mg} / \mathrm{dL}, P=0.12 ; 304 \mathrm{IU} / \mathrm{mL}$ vs $156 \mathrm{IU} / \mathrm{mL}, P=0.06$; $8555 / \mu \mathrm{L}$ vs $6666 / \mu \mathrm{L}, P=0.08$; and $6437 / \mu \mathrm{L}$ vs $4751 / \mu \mathrm{L}, P=0.10$, respectively). In the established RA group, only RF titer was found to be significant (189 IU/L vs $113 \mathrm{IU} / \mathrm{L}, P=0.04)$.

For visualization of association between those clinical biomarkers and patients, we used hierarchical clustering analysis (Figure 2A). The levels of IgA, neutrophil count, anti-CCP titers and RF titers were found to be higher in the clusters that contained more patients who achieved remission. IgA levels, antiCCP titer and neutrophil count were significantly correlated with the change of CDAl after 6 months with treatment by abatacept in patients with early RA $(R=-0.44, P=0.03 ; R=-0.56, P<0.01 ; R=-0.41, P=$ 0.04 , respectively), while RF significantly correlated with those with the established RA group $(R=-0.35, P$ $<0.01$ ) (Figure 2B). Receiver operating curve analysis demonstrated an optimal cut-off value of baseline serum IgA level, anti-CCP titer and neutrophil count to predict CDAl remission achievement in the early group as $342 \mathrm{mg} / \mathrm{dL}$ with sensitivity of $66.7 \%$, specificity of $86.7 \%$, and area under the curve (AUC) of $0.659,330 \mathrm{IU} / \mathrm{mL}$ with sensitivity of $44.4 \%$, specificity of $86.7 \%$ and AUC of 0.741 , and $6200 / \mu \mathrm{L}$ with sensitivity of $55.6 \%$, specificity of $86.7 \%$ and AUC of 0.704 , respectively (Figure $2 \mathrm{C}$ and $2 \mathrm{D}$ ). RF in the established group showed a cut-off of $212 \mathrm{IU} / \mathrm{mL}$ with sensitivity of $45 \%$, specificity of $88.1 \%$ and AUC of 0.622 .

Patients in the early RA group who satisfied with each cut-off value showed better response to abatacept treatment (Figure $2 \mathrm{E}-2 \mathrm{G}$ ). The higher the number of variables the early patients satisfied, the better response they showed to abatacept treatment (Supplementary Figure 1). Although patients in the established RA group with $\mathrm{RF} \geq 212 \mathrm{IU} / \mathrm{mL}$ responded well to abatacept treatment shortly after initiation of the treatment compared to those without the treatment, significance was found to be diminished at 6 months post-treatment (Figure $2 \mathrm{H}$ ). 
Immune-phenotyping and cytokine measurement of peripheral blood was conducted prospectively in 33 patients ( 9 early RA and 24 established RA). The characteristics of patients who were subjected to immune-phenotyping and cytokine analysis have been summarized in Supplementary Table 5. No major difference was found between patients with immune-phenotyping and those without immunephenotyping except for differences in the lymphocyte counts and a history of use of other biological agents. In the early RA group, the proportions of the activated Th17 cells (aTh17) and the activated Treg cells (aTreg) at baseline were found to be significantly higher in patients with remission compared to those without remission (aTh17/Th17, $2.9 \%$ vs $1.1 \%, P=0.02$; aTreg/Treg, $34.3 \%$ vs $17 \%, P=0.03$ ) (Table 1 and Figure 3A). Regarding the established RA group, no difference was found between the patients with remission and those without remission (Table 1).

To validate the importance of aTh17 and aTreg and to identify the patient subgroups in relation to these phenotypes, we screened the key features using the Cox regression model-related method [10]. Similar to the comparison between patients with remission and without remission, aTreg and aTh17 were selected as remission-related features in the early RA group, and three subgroups were identified based on the proportions of aTreg and aTh17 (group 1, $n=4$; group 2, n=2; group 3, $n=3$ ). We observed that all, one and none of the patients in the groups 1, 3 and 2 achieved remission, respectively (Figure 3B). Although the consensus matrix appeared to be varied among the patients of group 1 compared to matrices of other groups, the Silhouette width values supported the validity of the process of making subgroups using aTreg and aTh17 as all the patients had positive silhouette width (Figure 3C and 3D). Principal component analysis using aTreg and aTh17 suggested each subgroup had different characteristics (Figure 3E). Hierarchical clustering analysis using all parameters showed heterogeneity in the cell subpopulations and cytokine profiling except for aTreg and aTh17 (Figure 3F). When focused on correlation with change in disease activity, only the proportion of aTh17 showed significant correlation with CDAl improvement $(R=-0.7, P=0.035)$ (Figure 3F and 3G).

We performed the same analysis with the longitudinal data from patients at pre-treatment stage to 6 months post-treatment stage in the early RA group. The total CD4 T cells and naïve CD 4 T cells (NCD4) populations significantly increased within 6 months in patients with remission compared to those without remission ( $5 \%$ vs $-6 \%, P=0.02 ; 14.7 \%$ vs $-10.9 \%, P=0.02$, respectively), while the population of effector CD4 T cells (ECD4) and activated Th2 cells (aTh2) significantly decreased in the patients with remission compared to those without remission $(-29.2 \%$ vs $11.7 \%, P=0.01 ;-59.1 \%$ vs $-5.9 \%, P=0.03$, respectively) (Table 2).

During the process of feature selection based on the Cox regression model, the changes in the total CD4 T cells, NCD4, ECD4, aTreg, aTh2 and CD86 $\mathrm{B}$ cells were identified as remission-related features. The patients were divided into three subgroups, and the patients in group 1 achieved remission earlier than 
those in group 2 (Figure $3 \mathrm{H})$. Notably, the data from all three patients in this group 1 overlapped with the pre-treatment data of patients in group 1. The consensus matrix, the Silhouette width and the principal component analysis demonstrated that the three subgroups were clearly distinguishable (Figure $3 \mathrm{I}, 3 \mathrm{~J}$ and 3K). The heat-maps show that the total CD4 T cells, in particular NCD4, increased after treatment in group 1, and the ECD4 T cells decreased after treatment in groups 1 and 2 (Figure 3L). Regarding the correlation with disease activity, the changes in the total CD4 cells, NCD4 and NCD8 T cells, CD86 ${ }^{+} \mathrm{B}$ cells, ECD4, and aTh2 correlated with CDAl improvement $(R=-0.88, P<0.01 ; R=-0.87, P<0.01 ; R=-0.7, P$ $=0.04 ; R=-0.72, P=0.04 ; R=0.87, P<0.01 ; R=0.72, P=0.03$, respectively) (Figure $3 \mathrm{~L}$ ). However, comparison of these parameters among patients with remission and those without remission throughout the 6 months duration after abatacept treatment initiation, levels of NCD8 and CD86 ${ }^{+}$B cells fluctuated and there was no difference at 6 months post-treatment (Figure 3M and Table 2). In accordance with the cell-surface markers considered for this study, the increase in the levels of NCD4 may reflect a decrease in the levels of ECD4.

Although the patients in the established RA group were divided into six subgroups using the pre-treatment data set and four groups using the longitudinal data set, both the Cox regression models were found to be insignificant (Supplementary Figure 2A and 2F), and the patient subgroups using selected features were not observed to make clusters in the principal component analysis (Supplementary Figure 2D and 2I). Only lower IFN-y levels before treatment was associated with better CDAI improvement (Figure 4A and Supplementary Figure 2E).

In summary, the comparison between remission group and the non-remission group showed that aTh17 levels and aTreg levels before treatment and decrease in the levels of ECD 4 and aTh2 may be used as biomarkers for evaluating the response to abatacept treatment in the early RA group. Using the Cox regression model-related method, we internally validated their efficacy to distinguish between the feature of remission and non-remission, and we found subgroups of patients based on remission-related features. In the established RA group, only IFN- $y$ before treatment was associated with response to abatacept treatment.

\section{Association among key features in the early RA group}

We investigated association between clinical biomarkers (Figure 2), cell subpopulations, and cytokines by visually analyzing the similarity between these features using multi-dimensional scaling plots in the early RA group (Figure 3N). Using pre-treatment data, anti-CCP titer was found to be in the positive MDS1 axis and in the negative MDS2 axis along with cytokines and functional T cell subpopulations including aTh17 and aTreg, while IgA levels and neutrophil counts were in a different cluster. MDS plot by longitudinal datasets also demonstrated that IgA levels were separated from remission-related key cell subpopulations, suggesting that IgA levels may be an independent biomarker for peripheral cell subpopulations and for cytokines. Interestingly, neutrophil count was close to the change in the levels of 
NCD4 and on the opposite side of the levels of ECD4, indicating that the change in the NCD4/ECD4 ratio was associated with the neutrophil count.

\section{Correlation between IFN-y and activated CD56 bright $N K$ cells}

We had previously reported the association between inadequate response to abatacept treatment with the population of NK cells and specific-CD56 signature genes [17]. As NK cells, particularly the CD56 bright subset, are potent IFN- $\gamma$ producers, we hypothesized that the concentration of IFN- $\gamma$ in the established RA group may be associated with the NK cell population. To confirm this, we additionally analyzed the NK cell subpopulations in the patients with established RA (Figure 4). Our analysis found that the IFN-y levels significantly correlated with the NKG2D ${ }^{+} \mathrm{CD} 56^{\text {bright }} \mathrm{NK}$ cells, which is the activated CD $56^{\text {bright }} \mathrm{NK}$ cell subpopulation $\left(\mathrm{R}=0.43, P=0.04\right.$ ) (Figure $4 \mathrm{~B}$ and $4 \mathrm{C}$ ). In addition, decrease in the NKG2D ${ }^{+}$CD56 ${ }^{\text {bright }} \mathrm{NK}$ cell population after abatacept treatment was found to be significantly larger in patients with remission compared to those without remission, while no difference was seen in any other NK cell subpopulations $(-10.1 \%$ vs $-2 \%, P=0.03)$ (Figure $4 \mathrm{D}$ ).

\section{Discussion}

This study identified that high anti-CCP titers, serum IgA levels and neutrophil counts at the time of abatacept treatment initiation were predictive clinical biomarkers for achievement of remission after abatacept therapy in patients with seropositive early RA. In the immune-phenotyping analysis, the proportion of aTh17 and aTreg before abatacept treatment initiation was found to be higher in patients who achieved remission, and the improvement in disease activity correlated with a decrease in the effector helper T lymphocytes. Multi-dimensional scaling analysis revealed association pairs among antiCCP titers, aTh17 and aTreg, and neutrophils and changes in the ECD4 levels, while IgA was found to be independent of any association. In contrast, in case of patients with established RA, RF titers showed a weak performance in predicting the response to abatacept treatment. At the same time, lower levels of IFN-y before treatment was associated with better response to abatacept treatment.

Our study indicated that abatacept works by suppressing the development of the effector helper $\mathrm{T}$ cells, and anti-CCP titer is a predictive factor for response to abatacept treatment $[1,6]$. In RA-specific immune reaction, it is hypothesized that exposure of mucosal surfaces, such as the lungs and the intestines that are the main sources of anti-CCP antibodies, Th17 and Treg, to environmental agents triggers the development of RA [18-20]. Indeed, Th17 cells have been found to be increased in the early stages of RA concomitant with the disease activity [21], driving chronic destructive inflammation [22]. Although the extent to which Th17 contributes to anti-CCP antibody production is unknown, its ability to help the $B$ cells has been confirmed in mice models $[23,24]$. However, there have been conflicting reports demonstrating a poor association with peripheral Th17 subpopulation and anti-CCP antibody in early RA [25]. The possible explanation for this conflict may be that Th17 proportions measured in the previous 
reports consisted of the memory Th17, and not proportions of the activated Th17. Taking this into consideration, the magnitude of activation of the Th17 cells may be important for the autoimmune response. In reality, the multi-dimensional scaling plots in the current study showed that the total functional T cell subpopulations and their activated populations clustered separately.

The proportion of activated Treg cells was also associated with response to abatacept treatment in early RA. However, the function of Treg in RA is impaired by inflammatory cytokines, and the tumor necrosis factor a-induced Treg dysfunction correlates with pathogenic Th17 and Th1 in the synovium of RA patients [26]. It has been demonstrated that abatacept treatment improved Treg function in patients with RA who responded well based on the ACR50 response [27]. Nevertheless, larger cohort studies will be needed to explore the mechanism of restoration of Treg function by abatacept and its association with the efficacy of the treatment.

$\lg \mathrm{A}$, which has been found as a predictive marker for evaluating the effectiveness of abatacept treatment in our study, is a type of immunoglobulin produced from class-switched B cells with the help of helper $T$ cells, and has a critical function in neutralizing microbial toxins and pathogens on mucosal sites [28]. IgA-related immune response including the IgA-isotype of RF and anti-CCP antibody occurs in individuals who are at a high risk of RA development and this event precedes the IgM- or IgG-RF and anti-CCP response with local mucosal inflammation [29-33]. This lag phase in the immune response may be the reason for the separate cluster of IgA levels that was formed from the anti-CCP titer and other key subpopulations in the multi-dimensional scaling plots.

Besides adaptive immunity markers, our study found neutrophils as a possible biomarker in early RA. It is well-known that neutrophils play a critical role in persistent inflammation and progression of joint damage in RA [32]. In addition, neutrophils may contribute to the development of RA through the formation of neutrophil extracellular traps (NETs), triggering T cell-mediated response and anti-CCP production in the lungs [34]. Although there have been no reports on the association between increased neutrophil levels and the therapeutic response to abatacept in RA, this association has been indicated in other autoimmune diseases such as in systemic lupus erythematosus [35]. Co-stimulatory molecules including CD80/CD86 co-localize in the NETs, suggesting that the interaction between T cells and neutrophil may also be a target for abatacept [36]. This is supported by the association between neutrophils and changes in the NCD4/ECD4 balance observed in the current study.

Interestingly, the useful biomarkers described above were only detected in patients with early RA, not in established RA. While naïve T cells require co-stimulation from CD80/86 on the dendric cells found in the lymphoid tissues, the activation of memory $T$ cells, that have been primed previously, has been found to be less dependent on co-stimulation than their dependency on naïve T cells [37]. Considering the fact that cumulative exposure to antigens promotes the accumulation of memory $T$ cells, it may be a reason why abatacept works better in patients with shorter disease duration [7]. In fact, a trial suggested the possibility that abatacept may alter the progression of RA at a very early stage, although it was not found to be statistically significant [38]. To confirm this, a larger randomized trial is in process [39]. 
In the established RA group, the patients with higher concentration of IFN- $y$ before treatment were found to be resistant to abatacept treatment. In addition, IFN-y levels significantly correlated with the activated CD56 bright NK cells, whose decrease was found to be substantially larger in patients who achieved remission compared with those who did not achieve remission. NK cells are considered to be one of the major players in autoimmune diseases, including RA [40,41]. The NK cells in the synovial fluid of patients with progressive active RA are potent producers of cytokines such as IFN-y [42]. Previously, by using whole transcriptome analysis, we reported that NK cell-related genes were significant predictors of inadequate response to abatacept treatment [17]. Calcineurin inhibitors have been reported as possible inhibitors for NK cells including their cytokine-producing subpopulations [43-45], implying that they may be attractive drugs for patients that do not respond to abatacept treatment.

Several limitations to our study should be noted. First, the number of patients was small, especially those with early stage RA. In Japan, there is an empirical tendency to presume that there is a low risk of developing serious infections with abatacept treatment as compared to treatment with other antirheumatic drugs in elderly patients, who frequently overlap with patients with established RA [46]. Second, immune-phenotyping analysis was not conducted in all the patients, and usage of cryopreserved PBMCs may decrease the reliability of the methods [47]. Third, more than half of the patients with immune-phenotyping analysis were treated with concomitant methotrexate, which may skew immunephenotyping and cytokine profiles [2]. Fourth, our analysis did not check other T cell subpopulations, which are also players in the adaptive immunity and involved in the pathogenesis of RA, such as the the follicular helper T cells and peripheral T helper cells $[48,49]$. Fifth, we used peripheral blood and not synovium. Although there may be a difference in the biomarkers present in the blood and in the synovium, we suggest that blood samples are appropriate as the purpose of the study was to screen for useful biomarkers for evaluating the response to abatacept treatment, and not to explore the mechanism of action of abatacept. Notably, a decrease in the levels of aTh2 was identified as a remission-related marker in the early RA group, while the aTh2-response demonstrated inhibitory effects in the synovium of the RA patients, implying a change in the aTh2 level reflects the extent of the patients' responses to abatacept treatment [50].

\section{Conclusions}

Our study showed that serum IgA levels, anti-CCP titer and neutrophil counts were predictive biomarkers for the response to abatacept in patients with early, seropositive RA. Immune-phenotyping analysis suggests that these biomarkers except for IgA may be associated with the activated effector helper T cell subsets, which may be inhibited by abatacept treatment. In the established RA patients, inhibition in the production of IFN-y and the number of CD $56^{\text {bright }} \mathrm{NK}$ cells may be the possible mechanism for clinical response.

\section{Abbreviations}


aTh1, activated Th1; aTh17, activated Th17; aTh2, activated Th2; aTreg, activated regulatory T cell; CCP, citrullinated peptide; CDAl, clinical disease activity score; $\mathrm{CM}$, classical monocyte; CMCD4, central memory CD4 T cell; CMCD8, central memory CD8 T cell; CRP, C-reactive protein; CTLA4, cytotoxic Tlymphocyte-associated antigen 4; ECD4, effector CD4 T cell; ECD8, effector CD8 T cell; EGA, evaluator global assessment; EMCD4, effector memory CD4 T cell; EMCD8, effector memory CD8 T cell; ESR, erythrocyte sedimentation rate; HAQ-DI, Health Assessment Questionnaire Disability Index; Ig, immunoglobulin; MB, memory B cell; MDS, multi-dimensional scaling; MMP-3, matrix metalloproteinase-3; MTreg, memory regulatory T cell; NB, naive B cell; NCD4, naive CD4 T cell; NCD8, naive CD8 T cell; NCM, non-classical monocyte; NETs, neutrophil extracellular traps; NONREM, non-remission; PGA, patient global assessment; RA, rheumatoid arthritis; REM, remission; RF, rheumatoid factor; SJC, swollen joint count; TJC, tender joint count; Treg, regulatory T cell. VAS, visual analogue scale; WBCs, white blood cells.

\section{Declarations}

Ethics approval and consent to participate: Ethics approval was obtained from the Institutional Review Board of Keio University School of Medicine.

Consent for publication: Not required.

Availability of data and materials: All custom computer codes in the generation or processing of the described data are available upon reasonable request.

Competing interests: J.I has declared no conflicts of interest. Y.K. has received grants or speaking fees from AbbVie, Astellas, Ayumi, Bristol-Myers, Chugai, Eisai, Eli Lilly, Hisamitsu, Jansen, Kissei, Pfizer, Sanofi, Takeda, Mitsubishi Tanabe, and UCB. J.K. has received grants or speaking fees from Astellas, Bristol-Myers, Chugai, Eisai, and Janssen. T.T. has received research grants or speaking fees from AbbVie, Asahikasei, Astellas, Astra Zeneca, Bristol-Myers, Chugai, Daiichi Sankyo, Eli Lilly, Janssen, Mitsubishi Tanabe, Novartis, Nipponkayaku, Taiho, Takeda, Teijin, and Pfizer.

Funding: Nothing to declare.

Authors' Contributions: Conceptualization: JI, JK and YK. Funding acquisition: TT. Data acquisition: JI and JK. Formal analysis: Jl. Writing and original draft preparation: Jl. Writing review and editing: YK and TT.

Acknowledgments: Nothing to declare.

\section{References}

1. Moreland L, Bate G, Kirkpatrick P. Abatacept. Nature Rev. Drug Discov 2006;5: 185-186.

2. Takeshita M, Suzuki K, Kondo Y, et al. Multi-dimensional analysis identified rheumatoid arthritisdriving pathway in human T cell. Ann Rheum Dis. 2019;78: 1346-1356. 
3. Reiser H., Stadecker, M. J. Costimulatory B7 molecules in the pathogenesis of infectious and autoimmune diseases. Engl. J. Med. 1996;335: 1369-1377.

4. Oryoji K, Yoshida K, Kashiwado Y, Tanaka K, Mizuki SI, Tsukamoto H, Kamada K, Akashi K. Shared epitope positivity is related to efficacy of abatacept in rheumatoid arthritis. Ann Rheum Dis 2018;77: 1234-1236.

5. Gottenberg JE, Courvoisier DS, Hernandez MV, lannone F, Lie E, Canhão H, Pavelka K, Hetland ML, Turesson C, Mariette X, Finckh A. Brief report: association of rheumatoid factor and anti-citrullinated protein antibody positivity with better effectiveness of abatacept: results from the Pan-European registry analysis. Arthritis Rheumatol 2016;68: 1346-1352.

6. Sokolove J, Schiff M, Fleischmann R, Weinblatt ME, Connolly SE, Johnsen A, Zhu J, Maldonado MA, Patel S, Robinson WH. Impact of baseline anti-cyclic citrullinated peptide-2 antibody concentration on efficacy outcomes following treatment with subcutaneous abatacept or adalimumab: 2-year results from the AMPLE trial. Ann Rheum Dis 2016;75: 709-714.

7. Harrold LR, Litman HJ, Connolly SE, Kelly S, Hua W, Alemao E, Rosenblatt L, Rebello S, Kremer JM. A window of opportunity for abatacept in RA: is disease duration an independent predictor of low disease activity/remission in clinical practice? Clin Rheumatol. 2017;36: 1215-1220

8. Aletaha D, Neogi T, Silman AJ, Funovits J, Felson DT, Bingham CO III, et al. 2010 rheumatoid arthritis classification criteria: an American College of Rheumatology/European League Against Rheumatism collaborative initiative. Arthritis Rheum 2010;62: 2569-81.

9. Knevel R, Lukas C, van der Heijde D, Rincheval N, Combe B, van der Helm-van Mil AH. Defining erosive disease typical of RA in the light of the ACR/EULAR 2010 criteria for rheumatoid arthritis; results of the data driven phase. Ann Rheum Dis. 2013;72(4):590-5

10. Aletaha D, Smolen J. The Simplified Disease Activity Index (SDAI) and the Clinical Disease Activity Index (CDAI): a review of their usefulness and validity in rheumatoid arthritis. Clin Exp Rheumatol. 2005;23(5 Suppl 39): S100-8.

11. Xu T, Le TD, Liu L, et al. CancerSubtypes: R/Bioconductor package for molecular cancer subtype identification, validation and visualization. Bioinformatics. 2017;33: 3131-3133.

12. Monti, Stefano, et al. Consensus clustering: a resampling-based method for class discovery and visualization of gene expression microarray data. Machine learning 2003;52: 91-118.

13. Wang, Bo, et al. Similarity network fusion for aggregating data types on a genomic scale. Nature methods 2014;11: 333-337.

14. Rousseeuw P.J. Silhouettes: a graphical aid to the interpretation and validation of cluster analysis. J. Comput. Appl. Math. 1987;20: 53-65.

15. Gabriel, K. R. The biplot graphic display of matrices with application to principal component analysis. Biometrika, 1971;58: 453-467.

16. Michael C. Hout, Megan H. Papesh, and Stephen D. Goldinger. Multidimensional scaling. Wiley Interdiscip Rev Cogn Sci. 2013; 4: 93-103. 
17. Nakamura S, Suzuki K, lijima $\mathrm{H}$, et al. Ident ification of baseline gene expression signatures predicting therapeutic responses to three biologic agents in rheumatoid arthritis: a retrospective observational study. Arthritis Res Ther. 2016;18: 159.

18. Malmström V, Catrina Al, Klareskog L. The immunopathogenesis of seropositive rheumatoid arthritis: from triggering to targeting. Nat Rev Immunol. 2017;17: 60-75.

19. Atarashi K, Tanoue T, Shima T, Imaoka A, Kuwahara T, Momose $Y$, et al. Induction of colonic regulatory T cells by indigenous Clostridium species. Science 2011;331: 337-41.

20. Lucchino B, Spinelli FR, lannuccelli C, et al. Mucosa-Environment Interactions in the Pathogenesis of Rheumatoid Arthritis. Cells. 2019;8(7).

21. Leipe J, Grunke M, Dechant C, Reindl C, Kerzendorf U, et al. Role of Th17 cells in human autoimmune arthritis. Arthritis Rheum 2010;62: 2876-2885.

22. van Hamburg JP, Asmawidjaja PS, Davelaar N, Mus AM, Colin EM, et al. Th17 cells, but not Th1 cells, from patients with early rheumatoid arthritis are potent inducers of matrix metalloproteinases and proinflammatory cytokines upon synovial fibroblast interaction, including autocrine interleukin-17A production. Arthritis Rheum 2011;63: 73-83.

23. Hickman-Brecks CL, Racz JL, Meyer DM, et al. Th17 cells can provide B cell help in autoantibody induced arthritis. J Autoimmun. 2011;36: 65-75.

24. Mitsdoerffer $M$, Lee $Y$, Jäger $A$, et al. Proinflammatory $T$ helper type 17 cells are effective B-cell helpers. Proc Natl Acad Sci U S A. 2010;107: 14292-7.

25. Arroyo-Villa I, Bautista-Caro M-B, Balsa A, et al. Frequency of Th17 CD4+ T Cells in Early Rheumatoid Arthritis: A Marker of Anti-CCP Seropositivity. PLoS ONE 2012;7: e42189.

26. Nie H, Zheng Y, Li R, et al. Phosphorylation of FOXP3 controls regulatory T cell function and is inhibited by TNF-alpha in rheumatoid arthritis. Nat Med 2013;19::322-810.

27. Álvarez-Quiroga C, Abud-Mendoza C, Doníz-Padilla L, et al. CTLA-4-Ig therapy diminishes the frequency but enhances the function of Treg cells in patients with rheumatoid arthritis. $J$ Clin Immunol. 2011;31: 588-95.

28. Cerutti A. The regulation of IgA class switching. Nat Rev Immunol. 2008;8: 421-34.

29. Kokkonen H, Mullazehi M, Berglin E, Hallmans G, Wadell G, Rönnelid J, Rantapää-Dahlqvist S. Antibodies of IgG, IgA and IgM isotypes against cyclic citrullinated peptide precede the development of rheumatoid arthritis. Arthritis Res Ther. 2011;13: R13.

30. Rantapää-Dahlqvist S, de Jong BA, Berglin E, et al. Antibodies against cyclic citrullinated peptide and IgA rheumatoid factor predict the development of rheumatoid arthritis. Arthritis Rheum 2003;48: 2741-9.

31. Ärlestig L, Mullazehi M, Kokkonen $\mathrm{H}$, et al. Antibodies against cyclic citrullinated peptides of IgG, IgA and IgM isotype and rheumatoid factor of IgM and IgA isotype are increased in unaffected members of multicase rheumatoid arthritis families from northern Sweden. Ann Rheum Dis. 2012;71: 825-829. 
32. K. Demoruelle, K. K. Harrall, L. Ho et al. Anti-citrullinated protein antibodies are associated with neutrophil extracellular traps in the sputum in relatives of rheumatoid arthritis patients. Arthritis Rheum 2017; 69:1165-1175.

33. Willis, V. C. et al. Sputa autoantibodies in patients with established rheumatoid arthritis and subjects at-risk for future clinically apparent disease. Arthritis Rheum. 2013;65: 2545-2554.

34. Wright HL, Moots RJ, Bucknall RC, Edwards SW. Neutrophil function in inflammation and inflammatory diseases. Rheumatology (Oxford). 2010;49: 1618-31.

35. Bandyopadhyay S, Connolly SE, Jabado O, et al. Identification of biomarkers of response to abatacept in patients with SLE using deconvolution of whole blood transcriptomic data from a phase Ilb clinical trial. Lupus Sci Med. 2017;28;4: e000206.

36. Rodriguez FM, Novak ITC. Costimulatory Molecules CD80 and CD86 Colocalized in Neutrophil Extracellular Traps (NETs). J Immunol Infect Dis 2016;3: 103.

37. Sprent J, Surh CD. T cell memory. Annu Rev Immunol. 2002;20:551-79.

38. Emery P, Durez P, Dougados M, et al. Impact of T-cell costimulation modulation in patients with undifferentiated inflammatory arthritis or very early rheumatoid arthritis: a clinical and imaging study of abatacept (the ADJUST trial). Ann Rheum Dis. 2010;69: 510-516.

39. Al-Laith M, Jasenecova M, Abraham S, et al. Arthritis prevention in the pre-clinical phase of RA with abatacept (the APIPPRA study): a multi-centre, randomised, double-blind, parallel-group, placebocontrolled clinical trial protocol. Trials. 2019;20: 429.

40. Gur, C. et al. The activating receptor NKp46 is essential for the development of type 1 diabetes. Nat Immunol 2010;11: 121-128.

41. Shegarfi H, Naddafi F, Mirshafiey A. Natural killer cells and their role in rheumatoid arthritis: friend or foe? ScientificWorldJournal. 2012;2012: 491974.

42. Yamin R, Berhani O, Peleg $\mathrm{H}$, et al. High percentages and activity of synovial fluid NK cells present in patients with advanced stage active Rheumatoid Arthritis. Sci Rep. 2019;9: 1351.

43. Pradier A, Papaserafeim M, Li N, et al. Small-Molecule Immunosuppressive Drugs and Therapeutic Immunoglobulins Differentially Inhibit NK Cell Effector Functions in vitro. Front Immunol. 2019;10;10: 556.

44. Kim TJ, Kim N, Kang HJ, et al. FK506 causes cellular and functional defects in human natural killer cells. J Leukoc Biol. 2010;88: 1089-97.

45. Neudoerfl C, Mueller BJ, Blume C, et al. The Peripheral NK Cell Repertoire after Kidney Transplantation is Modulated by Different Immunosuppressive Drugs. Front Immunol. 2013;4: 46.

46. Yun H, Xie F, Delzell E, Levitan EB, Chen L, Lewis JD, Saag KG, Beukelman T, Winthrop KL, Baddley JW, Curtis JR. Comparative Risk of Hospitalized Infection Associated with Biologic Agents in Rheumatoid Arthritis Patients Enrolled in Medicare. Arthritis Rheumatol. 2016;68: 56-66.

47. Yokoyama, W. M., Thompson, M. L. and Ehrhardt, R. O. Cryopreservation and thawing of cells. Curr Protoc Immunol Appendix 3, 3 G. 
48. Verstappen GM, Meiners PM, Corneth OBJ, et al. Attenuation of Follicular Helper T Cell-Dependent B Cell Hyperactivity by Abatacept Treatment in Primary Sjögren's Syndrome. Arthritis Rheumatol. 2017;69: 1850-1861.

49. Rao DA, Gurish MF, Marshall JL, et al. Pathologically expanded peripheral T helper cell subset drives B cells in rheumatoid arthritis. Nature. 2017;542: 110-114.

50. Chen Z, Bozec A, Ramming A and Schett G. Anti-inflammatory and immune-regulatory cytokines in rheumatoid arthritis. Nat Rev Rheumatol. 2019;15: 9-17.

\section{Tables}

Table 1. Proportion of cytokines and cell subsets at baseline in patients with remission and non-remission. 
CDAI status at 6 months

Early RA

Established RA

\begin{tabular}{|c|c|c|c|c|c|c|}
\hline & NONREM & REM & & NONREM & REM & \\
\hline $\mathrm{n}$ & 4 & 5 & $\mathrm{p}$ & 19 & 5 & $\mathrm{p}$ \\
\hline GM-CSF, pg/mL & $0.57(1.13)$ & $0.06(0.13)$ & 0.35 & $1.84(4.88)$ & $0.58(1.08)$ & 0.58 \\
\hline $\mathrm{IFN} \gamma, \mathrm{IU} / \mathrm{mL}$ & $0.62(1.24)$ & $1.80(2.35)$ & 0.40 & $6.53(12.80)$ & $0.88(0.56)$ & 0.34 \\
\hline $\mathrm{TNF} \alpha, \mathrm{pg} / \mathrm{mL}$ & $4.28(2.20)$ & $5.00(2.11)$ & 0.64 & $7.10(4.94)$ & $6.28(4.08)$ & 0.74 \\
\hline IL1, pg/mL & $0.12(0.25)$ & $0.16(0.28)$ & 0.85 & $0.87(1.52)$ & $0.28(0.29)$ & 0.40 \\
\hline IL2, pg/mL & $0.40(0.80)$ & $0.32(0.46)$ & 0.85 & $1.76(4.78)$ & $0.07(0.16)$ & 0.45 \\
\hline IL6, pg/mL & $3.89(2.70)$ & $3.04(2.23)$ & 0.62 & $6.28(5.94)$ & $2.82(1.88)$ & 0.22 \\
\hline IL8, pg/mL & $\begin{array}{c}20.23 \\
(25.82)\end{array}$ & $5.83(2.54)$ & 0.25 & $\begin{array}{c}51.04 \\
(155.16)\end{array}$ & $14.20(17.15)$ & 0.61 \\
\hline IL10, pg/mL & $1.73(3.01)$ & $1.71(1.54)$ & 0.99 & $\begin{array}{c}42.33 \\
(156.13)\end{array}$ & $\begin{array}{c}79.26 \\
(172.46)\end{array}$ & 0.65 \\
\hline IL12p70, pg/mL & $4.97(9.94)$ & $2.08(2.58)$ & 0.55 & $\begin{array}{c}57.31 \\
(216.50)\end{array}$ & $\begin{array}{l}328.74 \\
(730.91)\end{array}$ & 0.16 \\
\hline B cells, (\%) & $3.28(1.55)$ & $6.60(2.72)$ & 0.07 & $4.35(3.32)$ & $12.46(18.98)$ & 0.08 \\
\hline $\begin{array}{c}\text { CD80(+) B cells, } \\
(\%)\end{array}$ & $\begin{array}{l}12.28 \\
(4.76)\end{array}$ & $\begin{array}{l}10.60 \\
(4.12)\end{array}$ & 0.59 & $9.30(5.60)$ & $9.58(3.81)$ & 0.92 \\
\hline $\begin{array}{l}\text { CD86(+) B cells, } \\
(\%)\end{array}$ & $\begin{array}{c}39.92 \\
(19.04)\end{array}$ & $\begin{array}{c}38.90 \\
(11.84)\end{array}$ & 0.92 & $\begin{array}{r}40.57 \\
(17.12)\end{array}$ & $38.10(15.52)$ & 0.77 \\
\hline NB, (\%) & $\begin{array}{c}60.27 \\
(10.44)\end{array}$ & $\begin{array}{r}62.46 \\
(11.29)\end{array}$ & 0.77 & $\begin{array}{c}66.29 \\
(12.43)\end{array}$ & $65.98(14.45)$ & 0.96 \\
\hline CD80(+) NB (\%) & $2.62(1.71)$ & $1.68(0.80)$ & 0.30 & $1.98(1.65)$ & $1.95(1.54)$ & 0.97 \\
\hline CD86(+) NB, (\%) & $\begin{array}{c}30.10 \\
(24.97)\end{array}$ & $\begin{array}{r}33.36 \\
(17.02)\end{array}$ & 0.82 & $\begin{array}{l}37.12 \\
(18.00)\end{array}$ & 35.08 (17.36) & 0.82 \\
\hline $\mathrm{MB},(\%)$ & $\begin{array}{l}39.73 \\
(10.44)\end{array}$ & $\begin{array}{r}37.54 \\
(11.29)\end{array}$ & 0.77 & $\begin{array}{c}33.72 \\
(12.43)\end{array}$ & $34.02(14.45)$ & 0.96 \\
\hline $\mathrm{CD} 80(+) \mathrm{MB},(\%)$ & 24.32 & 19.28 & 0.31 & $17.38(7.60)$ & $18.20(5.15)$ & 0.82 \\
\hline
\end{tabular}




\begin{tabular}{ccccccc}
\hline CD86(+) MB, (\%) & 44.42 & 42.82 & 0.85 & 46.34 & $45.60(13.78)$ & 0.93 \\
& $(12.16)$ & $(11.90)$ & & $(16.49)$ & & \\
\hline NCM, (\%) & 22.32 & 12.90 & 0.36 & 18.49 & $13.76(3.55)$ & 0.48 \\
& $(20.80)$ & $(6.02)$ & & $(14.54)$ & & \\
\hline CD80(+) NCM, & $0.25(0.19)$ & $0.22(0.13)$ & 0.79 & $0.18(0.18)$ & $0.91(1.97)$ & 0.11 \\
$(\%)$ & & & & & & \\
\hline CD86(+) NCM, & 99.65 & 98.40 & 0.29 & $99.28(0.73)$ & $98.22(2.92)$ & 0.15 \\
$(\%)$ & $(0.26)$ & $(2.15)$ & & & & \\
\hline CM, (\%) & 77.67 & 87.10 & 0.36 & 81.51 & $86.24(3.55)$ & 0.48 \\
& $(20.80)$ & $(6.02)$ & & $(14.54)$ & & \\
\hline
\end{tabular}

\begin{tabular}{|c|c|c|}
\hline CD80(+) CM, (\%) & $0.05(0.06)$ & 0.04 \\
\hline
\end{tabular}

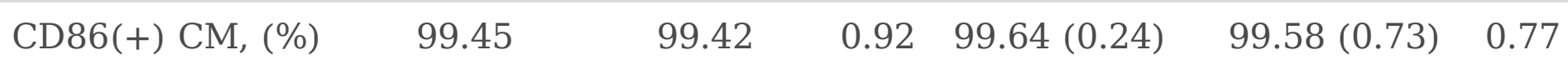
$(0.42) \quad(0.44)$

\begin{tabular}{ccccccc}
\hline CD4 T cells, (\%) & 48.36 & 52.36 & 0.42 & 50.21 & $50.71(13.81)$ & 0.95 \\
& $(8.20)$ & $(5.87)$ & & $(14.42)$ & & \\
\hline NCD4, (\%) & 40.55 & 42.52 & 0.83 & 41.15 & $46.32(17.13)$ & 0.52 \\
& $(14.33)$ & $(12.62)$ & & $(15.42)$ & & \\
\hline MCD4, (\%) & 55.30 & 64.04 & 0.33 & 58.23 & $55.70(11.34)$ & 0.79 \\
& $(16.42)$ & $(8.15)$ & & $(20.05)$ & & \\
\hline CMCD4, (\%) & 49.77 & 46.46 & 0.73 & 47.66 & $55.88(11.85)$ & 0.35 \\
& $(5.99)$ & $(17.14)$ & & $(18.14)$ & & \\
\hline EMCD4, (\%) & 50.23 & 53.54 & 0.73 & 52.34 & $44.12(11.85)$ & 0.35 \\
& $(5.99)$ & $(17.14)$ & & $(18.14)$ & & \\
\hline ECD4, (\%) & 16.57 & 19.64 & 0.83 & 19.04 & $11.24(12.66)$ & 0.29 \\
& $(23.10)$ & $(18.34)$ & & $(14.82)$ & & \\
\hline CD8 T cells, (\%) & 30.46 & 22.95 & 0.38 & $20.07(9.57)$ & $19.42(2.84)$ & 0.88 \\
& $(13.35)$ & $(10.62)$ & & & & \\
\hline NCD8 (\%) & $5.78(4.33)$ & $5.90(7.18)$ & 0.98 & $5.13(3.93)$ & $6.66(4.90)$ & 0.47 \\
\hline
\end{tabular}




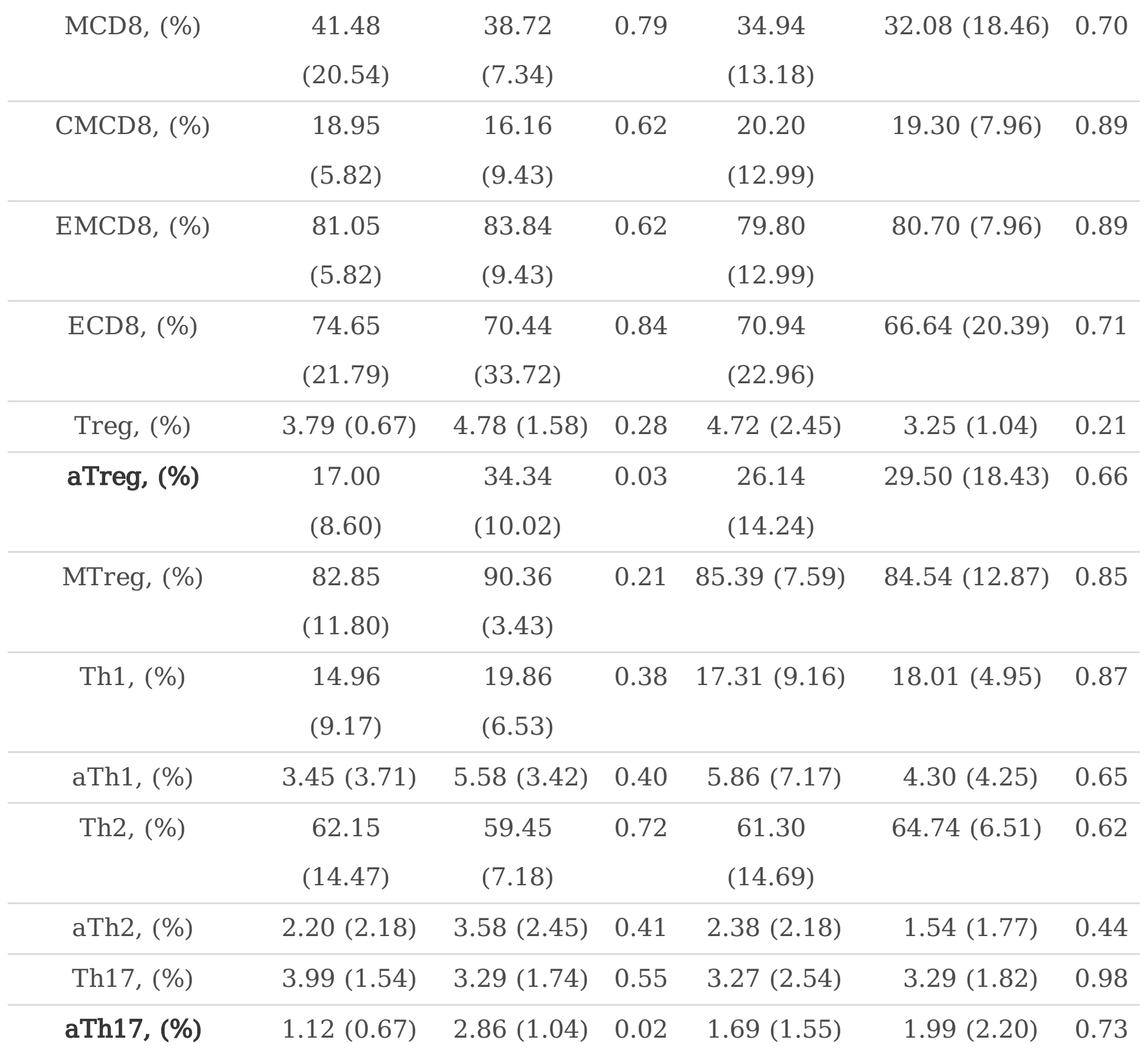

The value is a mean (standard deviation) unless otherwise stated.

aTh1, activated Th1; aTh2, activated Th2; aTh17, activated Th17; aTreg, activated regulatory T cell; CM, classical monocyte; CMCD4, central memory CD4 T cell; CMCD8, central memory CD8 T cell; ECD4, effector CD4 T cell; ECD8, effector CD8 T cell; EMCD4, effector memory CD4 T cell; EMCD8, effector memory CD8 T cell; GM-CSF, granulocyte macrophage colony-stimulating factor; IFN, interferon; IL, interleukin; MB, memory B cell; MTreg, memory regulatory T cell; NB, naive B cell; NCD4, naive CD4 T cell; NCD8, naive 
CD8 T cell; NCM, non-classical monocyte; TNF, tumor necrosis factor; Treg, regulatory T cell.

Table 2. Comparison of change of the proportion of cell subsets from baseline to 6 months between remission and non-remission. 
CDAI status at 6 months

\begin{tabular}{|c|c|c|c|c|c|c|}
\hline & \multicolumn{2}{|c|}{ Early RA } & \multicolumn{4}{|c|}{ Established RA } \\
\hline & NONREM & REM & & NONREM & REM & \\
\hline & 4 & 5 & $\mathrm{p}$ & 19 & 5 & $\mathrm{p}$ \\
\hline \multirow[t]{2}{*}{$\Delta \mathrm{TNF} \alpha, \mathrm{pg} / \mathrm{mL}$} & $-0.32(0.59)$ & $-0.43(0.90)$ & 0.85 & $-0.01(0.39)$ & 0.05 & 0.78 \\
\hline & & & & & $(0.45)$ & \\
\hline \multirow[t]{2}{*}{$\Delta \mathrm{IL} 6, \mathrm{pg} / \mathrm{mL}$} & $0.33(1.00)$ & $-0.72(2.30)$ & 0.43 & $-0.92(3.60)$ & 0.35 & 0.45 \\
\hline & & & & & $(0.07)$ & \\
\hline \multirow[t]{2}{*}{$\Delta \mathrm{IL} 8, \mathrm{pg} / \mathrm{mL}$} & $0.27(0.49)$ & $-0.78(1.22)$ & 0.15 & -4.70 & -0.73 & 0.52 \\
\hline & & & & $(13.29)$ & $(1.43)$ & \\
\hline \multirow[t]{2}{*}{$\Delta \mathrm{IL} 10, \mathrm{pg} / \mathrm{mL}$} & $-0.08(0.86)$ & $0.49(0.38)$ & 0.29 & $0.00(0.74)$ & 0.24 & 0.58 \\
\hline & & & & & $(1.22)$ & \\
\hline \multirow[t]{2}{*}{$\Delta \mathrm{B}$ cells, $(\%)$} & -16.80 & -28.87 & 0.59 & 6.14 & -2.03 & 0.89 \\
\hline & $(37.52)$ & $(26.22)$ & & $(124.08)$ & $(57.64)$ & \\
\hline \multirow{2}{*}{$\begin{array}{c}\Delta \text { CD80(+) B cells, } \\
(\%)\end{array}$} & 73.07 & 63.25 & 0.83 & 50.65 & 3.75 & 0.19 \\
\hline & $(78.47)$ & $(56.35)$ & & $(72.37)$ & $(50.43)$ & \\
\hline \multirow{2}{*}{$\begin{array}{c}\Delta \text { CD86(+) B cells, } \\
(\%)\end{array}$} & -25.54 & -11.04 & 0.08 & -3.12 & -12.37 & 0.44 \\
\hline & $(10.71)$ & $(10.07)$ & & $(24.27)$ & $(17.02)$ & \\
\hline \multirow[t]{2}{*}{$\Delta \mathrm{NB},(\%)$} & 6.08 & -5.78 & 0.43 & 2.80 & 12.78 & 0.45 \\
\hline & $(14.51)$ & $(24.83)$ & & $(27.71)$ & $(16.26)$ & \\
\hline \multirow[t]{2}{*}{$\Delta \mathrm{CD} 80(+) \mathrm{NB}(\%)$} & 219.33 & 98.22 & 0.38 & 135.07 & 13.90 & 0.14 \\
\hline & $(265.60)$ & $(110.79)$ & & $(169.21)$ & $(97.11)$ & \\
\hline \multirow{2}{*}{$\begin{array}{c}\Delta \mathrm{CD} 86(+) \mathrm{NB}, \\
(\%)\end{array}$} & 19.54 & -18.37 & 0.49 & 3.04 & -10.45 & 0.34 \\
\hline & $(116.25)$ & $(14.54)$ & & $(29.95)$ & $(7.81)$ & \\
\hline \multirow[t]{2}{*}{$\Delta \mathrm{MB},(\%)$} & -3.63 & 21.32 & 0.31 & 1.67 & -20.32 & 0.24 \\
\hline & $(19.34)$ & $(41.17)$ & & $(37.24)$ & $(30.42)$ & \\
\hline \multirow{2}{*}{$\begin{array}{c}\Delta \mathrm{CD} 80(+) \mathrm{MB} \\
(\%)\end{array}$} & 43.74 & 38.03 & 0.68 & 27.88 & 42.33 & 0.57 \\
\hline & $(27.63)$ & $(10.27)$ & & $(52.41)$ & $(38.78)$ & \\
\hline$\Delta \mathrm{CD} 86(+) \mathrm{MB}$ & $-15.28(7.84)$ & -15.50 & 0.97 & -12.73 & -9.24 & 0.79 \\
\hline
\end{tabular}




\begin{tabular}{|c|c|c|c|c|c|c|}
\hline$(\%)$ & & (10.35) & & $(21.42)$ & $(41.12)$ & \\
\hline \multirow[t]{2}{*}{$\triangle \mathrm{NCM},(\%)$} & -19.35 & 41.93 & 0.34 & 4.48 & -19.94 & 0.58 \\
\hline & $(50.48)$ & $(110.68)$ & & $(94.96)$ & $(29.68)$ & \\
\hline \multirow{2}{*}{$\begin{array}{c}\Delta \mathrm{CD} 80(+) \mathrm{NCM} \\
(\%)\end{array}$} & 96.67 & 250.00 & 0.45 & 77.07 & -27.04 & 0.38 \\
\hline & $(76.30)$ & (374.17) & & (252.98) & $(43.54)$ & \\
\hline \multirow{2}{*}{$\begin{array}{c}\Delta \mathrm{CD} 86(+) \mathrm{NCM}, \\
(\%)\end{array}$} & $0.13(0.22)$ & $0.63(1.10)$ & 0.40 & $0.36(0.83)$ & 0.26 & 0.81 \\
\hline & & & & & $(0.61)$ & \\
\hline \multirow[t]{2}{*}{$\Delta \mathrm{CM},(\%)$} & 24.67 & -1.95 & 0.28 & 9.48 & 3.85 & 0.68 \\
\hline & $(48.10)$ & (15.86) & & (29.73) & $(4.89)$ & \\
\hline \multirow{2}{*}{$\begin{array}{c}\Delta \mathrm{CD} 80(+) \mathrm{CM}, \\
(\%)\end{array}$} & $0.05(0.06)$ & $0.10(0.07)$ & 0.29 & $-0.03(0.07)$ & -0.12 & 0.09 \\
\hline & & & & & $(0.18)$ & \\
\hline \multirow{2}{*}{$\begin{array}{c}\Delta \mathrm{CD} 86(+) \mathrm{CM} \\
(\%)\end{array}$} & $0.33(0.32)$ & $0.16(0.55)$ & 0.61 & $-0.11(0.56)$ & 0.08 & 0.48 \\
\hline & & & & & $(0.32)$ & \\
\hline \multirow[t]{2}{*}{$\Delta \mathrm{CD} 4 \mathrm{~T}$ cells, $(\%)$} & $-6.00(5.80)$ & $5.03(4.88)$ & 0.02 & 1.09 & 4.60 & 0.74 \\
\hline & & & & (21.36) & $(17.05)$ & \\
\hline \multirow[t]{2}{*}{$\Delta \mathrm{NCD} 4,(\%)$} & -10.92 & 14.71 & 0.02 & 4.20 & 6.95 & 0.82 \\
\hline & $(13.26)$ & $(13.29)$ & & $(22.52)$ & $(27.18)$ & \\
\hline \multirow[t]{2}{*}{$\Delta \mathrm{MCD} 4,(\%)$} & -7.98 & -14.47 & 0.49 & -12.63 & -14.54 & 0.79 \\
\hline & $(16.70)$ & $(10.27)$ & & $(14.50)$ & $(11.86)$ & \\
\hline \multirow[t]{2}{*}{$\Delta \mathrm{CMCD} 4,(\%)$} & -1.70 & $6.25(7.75)$ & 0.26 & 7.20 & 4.22 & 0.77 \\
\hline & $(11.81)$ & & & $(21.44)$ & $(13.55)$ & \\
\hline \multirow[t]{2}{*}{$\triangle \mathrm{EMCD} 4,(\%)$} & 1.72 & $-5.96(6.41)$ & 0.23 & -8.02 & -3.45 & 0.62 \\
\hline & $(10.92)$ & & & $(16.97)$ & $(21.19)$ & \\
\hline \multirow[t]{2}{*}{$\Delta$ ECD4, (\%) } & 11.69 & -29.22 & 0.01 & -17.23 & 6.11 & 0.27 \\
\hline & $(19.25)$ & $(16.88)$ & & $(36.65)$ & $(57.50)$ & \\
\hline \multirow[t]{2}{*}{$\Delta$ CD8 T cells, $(\%)$} & -2.19 & 0.80 & 0.71 & -4.17 & 1.38 & 0.54 \\
\hline & $(11.43)$ & $(11.57)$ & & $(17.06)$ & $(20.11)$ & \\
\hline \multirow[t]{2}{*}{$\triangle \mathrm{NCD} 8(\%)$} & -22.78 & 7.87 & 0.05 & 10.57 & 18.87 & 0.63 \\
\hline & (15.83) & $(20.64)$ & & $(34.87)$ & $(30.31)$ & \\
\hline
\end{tabular}




\begin{tabular}{|c|c|c|c|c|c|c|}
\hline$\Delta \mathrm{MCD} 8,(\%)$ & $\begin{array}{c}-6.61 \\
(20.14)\end{array}$ & $\begin{array}{r}-10.62 \\
(12.03)\end{array}$ & 0.72 & $\begin{array}{c}1.49 \\
(34.66)\end{array}$ & $\begin{array}{c}-5.48 \\
(17.74)\end{array}$ & 0.67 \\
\hline \multirow[t]{2}{*}{$\triangle \mathrm{CMCD} 8,(\%)$} & -11.34 & -13.20 & 0.88 & 4.59 & -14.42 & 0.24 \\
\hline & $(21.47)$ & $(15.66)$ & & $(34.32)$ & $(11.86)$ & \\
\hline \multirow[t]{2}{*}{$\triangle \mathrm{EMCD} 8,(\%)$} & $1.97(5.42)$ & $3.86(4.63)$ & 0.59 & $-2.08(6.55)$ & 3.84 & 0.07 \\
\hline & & & & & $(4.07)$ & \\
\hline \multirow[t]{2}{*}{$\Delta \mathrm{ECD8},(\%)$} & $4.14(2.98)$ & -4.77 & 0.27 & 3.43 & -7.16 & 0.48 \\
\hline & & $(14.57)$ & & (31.94) & $(12.54)$ & \\
\hline \multirow[t]{2}{*}{$\Delta$ Treg, (\%) } & -53.98 & -51.38 & 0.85 & -39.88 & -40.58 & 0.96 \\
\hline & $(19.91)$ & $(19.76)$ & & $(31.25)$ & $(25.06)$ & \\
\hline \multirow[t]{2}{*}{$\Delta$ aTreg, (\%) } & -25.56 & -53.90 & 0.06 & -29.67 & -45.41 & 0.34 \\
\hline & $(17.12)$ & $(19.57)$ & & $(33.07)$ & $(28.92)$ & \\
\hline \multirow[t]{2}{*}{$\Delta$ MTreg, (\%) } & $-3.31(8.78)$ & $-7.88(5.51)$ & 0.37 & $-1.80(4.60)$ & -5.76 & 0.11 \\
\hline & & & & & $(5.12)$ & \\
\hline \multirow[t]{2}{*}{$\Delta$ Th1, (\%) } & 16.93 & -11.84 & 0.59 & -14.57 & -20.14 & 0.77 \\
\hline & $(96.87)$ & $(52.62)$ & & $(41.30)$ & $(15.87)$ & \\
\hline \multirow[t]{2}{*}{$\Delta \mathrm{aTh} 1,(\%)$} & -20.86 & -60.26 & 0.35 & -2.50 & -17.09 & 0.86 \\
\hline & $(85.55)$ & $(23.44)$ & & (175.77) & $(48.04)$ & \\
\hline \multirow[t]{2}{*}{$\Delta \mathrm{Th} 2,(\%)$} & 7.45 & 14.19 & 0.48 & 15.31 & 9.77 & 0.56 \\
\hline & (12.03) & $(14.67)$ & & $(20.16)$ & (8.48) & \\
\hline \multirow[t]{2}{*}{$\Delta \mathrm{aTh} 2,(\%)$} & -5.88 & -59.08 & 0.03 & -6.09 & -17.07 & 0.75 \\
\hline & (37.55) & (18.28) & & (71.17) & $(55.25)$ & \\
\hline \multirow[t]{2}{*}{$\Delta \operatorname{Th} 17,(\%)$} & -9.62 & -18.67 & 0.70 & -25.84 & -32.43 & 0.68 \\
\hline & $(48.36)$ & $(15.96)$ & & (33.13) & $(24.59)$ & \\
\hline \multirow[t]{2}{*}{$\Delta \mathrm{aTh} 17,(\%)$} & -68.06 & -70.20 & 0.90 & -38.05 & -67.50 & 0.30 \\
\hline & $(28.46)$ & (20.07) & & (58.18) & $(41.31)$ & \\
\hline
\end{tabular}

The value is a mean (standard deviation) unless otherwise stated. 
aTh1, activated Th1; aTh2, activated Th2; aTh17, activated Th17; aTreg, activated regulatory T cell; CM, classical monocyte; CMCD4, central memory CD4 T cell; CMCD8, central memory CD8 T cell; ECD4, effector CD4 T cell; ECD8, effector CD8 T cell; EMCD4, effector memory CD4 T cell; EMCD8, effector memory CD8 T cell; IFN, interferon; IL, interleukin; MB; memory B cell; MTreg, memory regulatory T cell; NB, naive B cell; NCD4, naive CD4 T cell; NCD8, naive CD8 T cell; NCM, non-classical monocyte; TNF, tumor necrosis factor; Treg, regulatory $\mathrm{T}$ cell.

\section{Supplementary File Information}

Supplementary Figure 1. Time course of CDAl improvement. Time course of CDAl improvement according to the number of variables over than each cut-off value in patients with early RA. P-value by the Student's t-test : $p<0.05$ between patients in group 0 and group $2(\#), 1$ and $2(\mathbb{\nabla}), 0$ and $3(\mathbb{X})$ and 1 and $3(\mathbb{X})$ after using analysis of variance. Results have been represented as mean \pm standard deviation. The cut-off values for remission, low disease activity, moderate disease activity and high disease activity were as follows: $\mathrm{CDAl} \leq 2.8,2.8<\mathrm{CDAl} \leq 10,10<\mathrm{CDAl} \leq 22$ and $\mathrm{CDAl}>22$. CCP, cyclic citrullinated peptide; CDAl, clinical disease activity score; RA, rheumatoid arthritis.

\section{Supplementary Figure 2. Peripheral immune-phenotyping and cytokines in patients with established RA. A} and F) Cumulative incidence plot for remission achievement according to subgroups of patients derived from remission-related features derived from the pre-treatment dataset $(A)$ and the longitudinal dataset $(F)$. The effect of features upon the time to achieve remission was compared by the Cox regression model.

B and G, Heat-map of the patient similarity matrix by remission-related features derived from pretreatment dataset $(B)$ and longitudinal dataset $(G)$.

$\mathrm{C}$ and $\mathrm{H}$, the Silhouette width shows whether a patient has been matched to its identified subgroup compared to other subgroups derived from the pre-treatment dataset (C) and the longitudinal dataset $(H)$. A high value indicates that the patient has been well- matched. Each horizontal line represents a patient in the Silhouette plot. The length of the line represents the Silhouette width that the patient had.

D and I, the Principal component analysis by selected key features derived from the pre-treatment dataset (D) and the longitudinal dataset (I). In biplot, patients have been displayed as points while features are displayed as vectors.

$\mathrm{E}$ and $\mathrm{J}$, Hierarchical clustering analysis by all parameters of the pre-treatment dataset $(\mathrm{E})$ and the longitudinal dataset $(\mathrm{J})$. Bottom boxplot represents the proportion of CDAl improvement 6 months after abatacept initiation. Heatmap on the right of main one represents how each parameter correlates with the proportion of CDAl improvement by depth of color; ' ${ }^{\prime}$ ': $p<0.05$ by the Spearman's correlation test. 
aTh1, activated Th1; aTh2, activated Th2; aTh17, activated Th17; aTreg, activated regulatory T cell; CCP, cyclic citrullinated peptide; CDAl, clinical disease activity score; $C M$, classical monocyte; CMCD4, central memory CD4 T cell; CMCD8, central memory CD8 T cell; ECD4, effector CD4 T cell; ECD8, effector CD8 T cell; EMCD4, effector memory CD4 T cell; EMCD8, effector memory CD8 T cell; MB; memory B cell; MTreg, memory regulatory T cell; NB, naive B cell; NCD4, naive CD4 T cell; NCD8, naive CD8 T cell; NCM, nonclassical monocyte; NONREM, non-remission; RA, rheumatoid arthritis; REM, remission; Treg, regulatory $T$ cell.

\section{Figures}

(A)

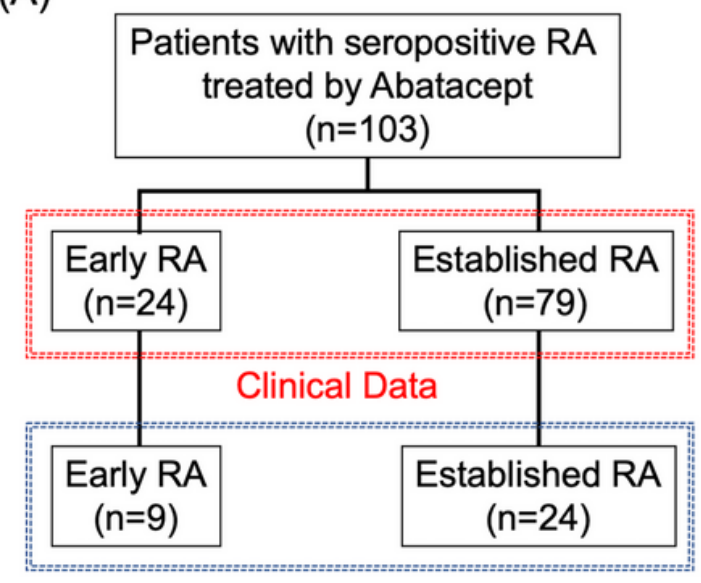

Clinical Data

$+$

Immunophenotyping

(week 0, 2, 12, 24)
(B)

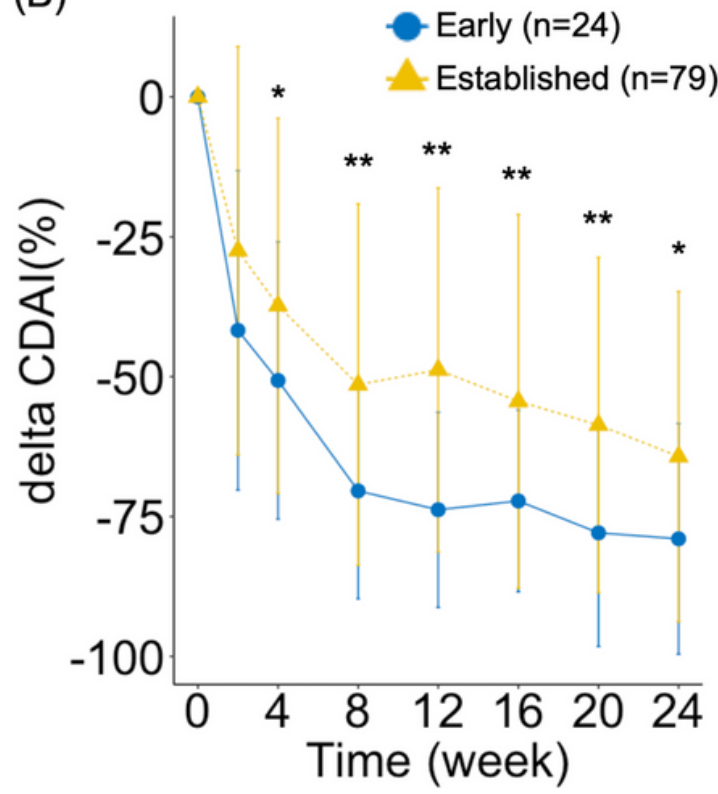

Early RA

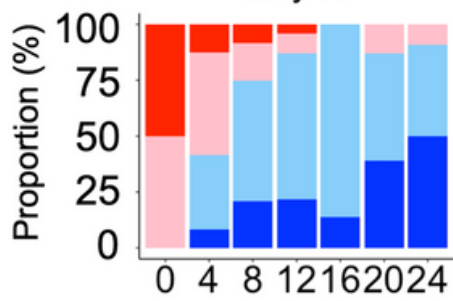

Established RA

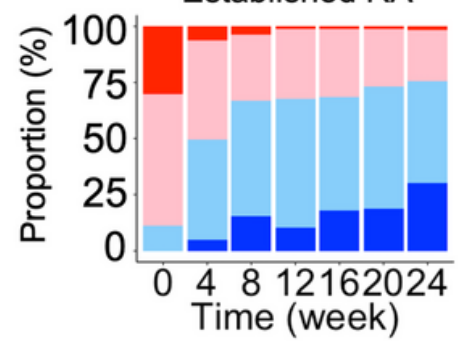

CDAl $>22 \quad 22 \geq \mathrm{CDAl}>10$

$10 \geq \mathrm{CDAl}>2.8 \square 2.8 \geq \mathrm{CDAl}$

\section{Figure 1}

Time course of CDAl improvement. A) Enrollment of patients in the current study. B) The proportion of CDAl improvement. The difference in CDAl improvement between patients with early and established RA was compared using the Student's t-test: ' $* \star^{\prime}: p<0.01$, ' $*$ ': $p<0.05$. Results have been represented as mean \pm standard deviation. The disease activity category has been shown according to CDAl. The cut-off values for remission, low disease activity, moderate disease activity and high disease activity were as follows: $\mathrm{CDAl} \leq 2.8,2.8<\mathrm{CDAl} \leq 10,10<\mathrm{CDAl} \leq 22$ and $\mathrm{CDAl}>22$. CDAl, clinical disease activity score; RA, rheumatoid arthritis. 
(A)

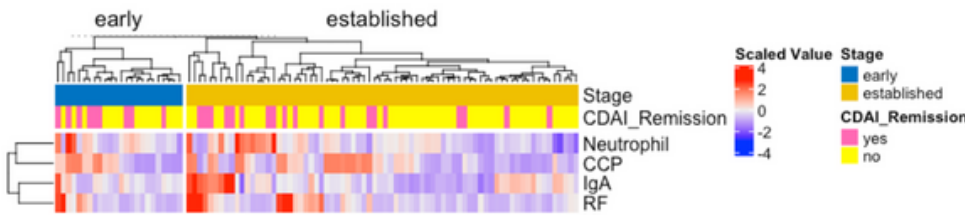

(B)

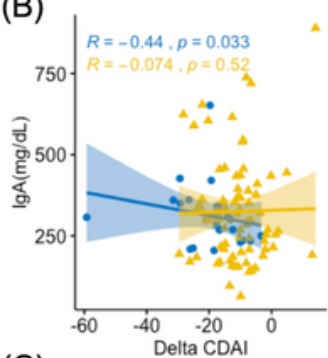

(C)

$\lg \mathrm{A}$

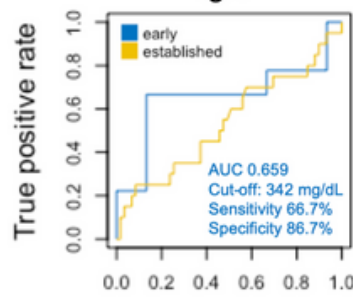

False positive rate
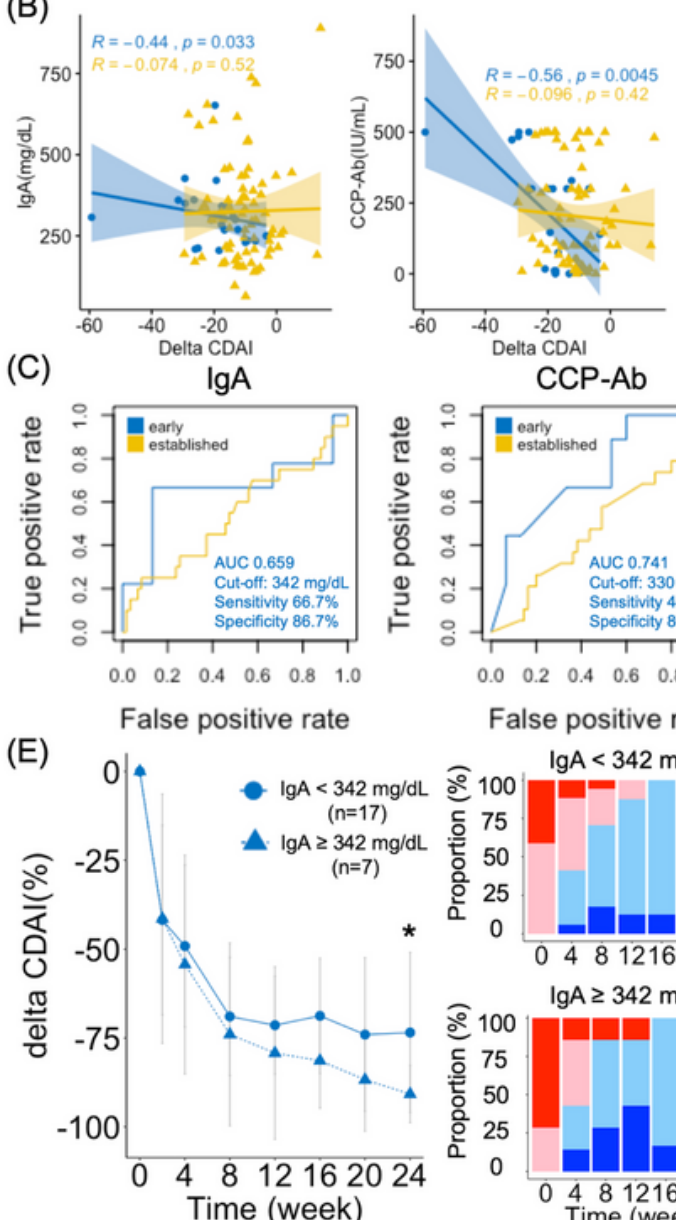

CCP-Ab

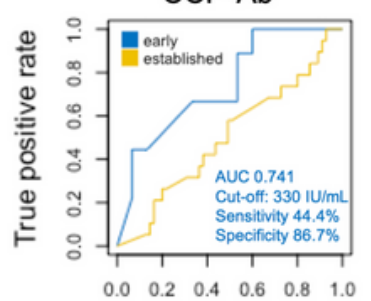

False positive rate

(G)

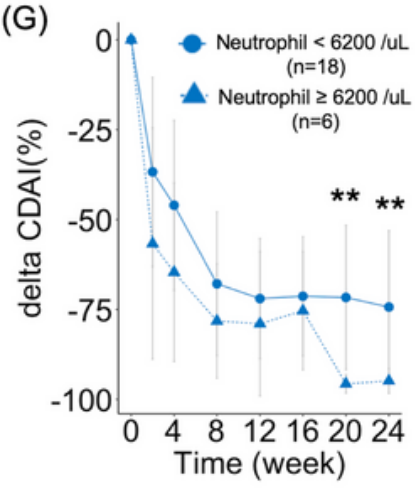

$\lg \mathrm{A}<342 \mathrm{mg} / \mathrm{dL}$

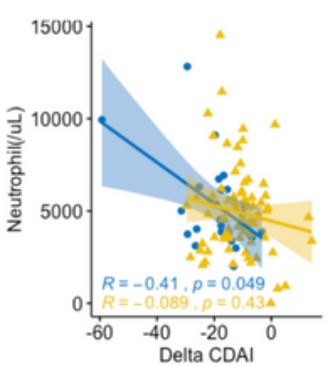

(H)

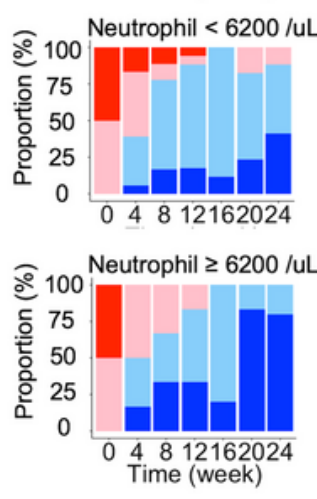

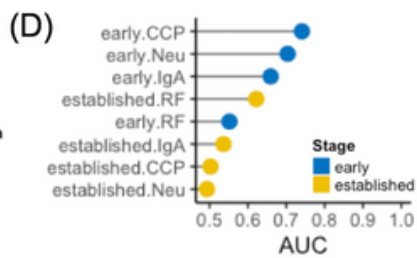

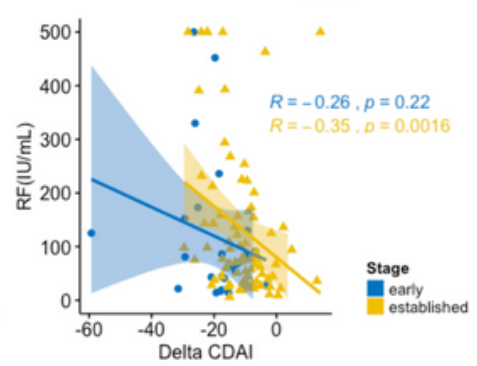

Neutrophil

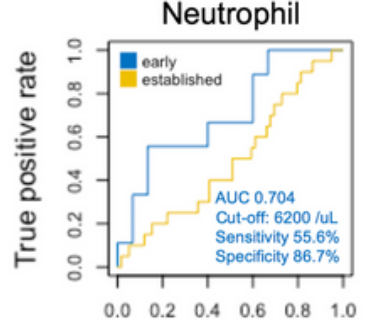

False positive rate

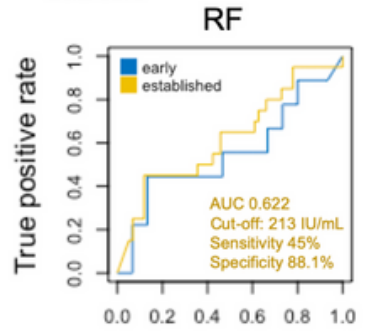

False positive rate
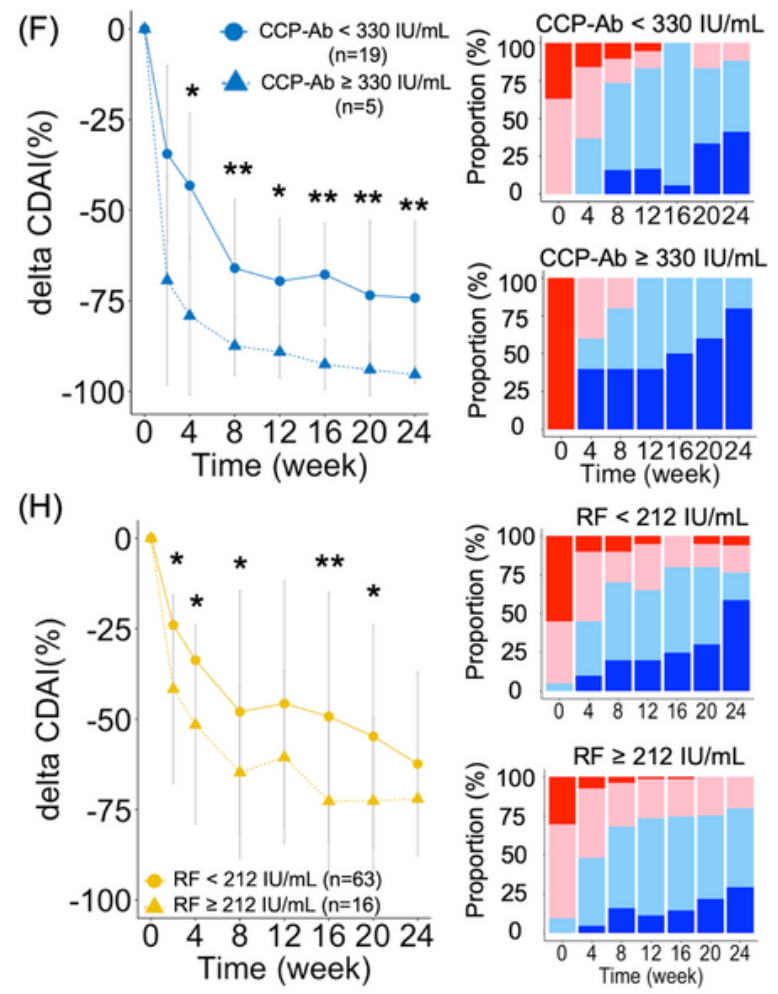

CDAl > $22 \quad 22 \geq \mathrm{CDAl}>10$

\section{Figure 2}

Predictive clinical biomarkers for response to abatacept. A) Hierarchical clustering analysis using laboratory tests. B) Correlation between laboratory tests and improvement of disease activity 6 months after abatacept treatment initiation. $C$ and D) Receiver operating characteristic curve analysis of serum IgA level, anti-CCP titer neutrophil count and RF titer for prediction of CDAl remission at 6 months.

Predictive performance has been shown by colored-text and curve; early RA by blue and established RA 
by gold. E-H) Time course of CDAl improvement according to cut-off values of IgA (E), anti-CCP titer (F) and neutrophil count $(G)$ in patients with early RA, and RF titer in patients with established RA $(H)$. P-value by the Student's t-test: '*k': $p<0.01,{ }^{\prime \prime \prime}: p<0.05$. Results have been represented as mean \pm standard deviation. The cut-off values for remission, low disease activity, moderate disease activity and high disease activity were as follows: $\mathrm{CDAl} \leq 2.8,2.8<\mathrm{CDAl} \leq 10,10<\mathrm{CDAl} \leq 22$ and $\mathrm{CDAl}>22$. ACPA, anticyclic citrullinated peptide antibody; AUC, area under the curve; RA, rheumatoid arthritis; RF, rheumatoid factor.
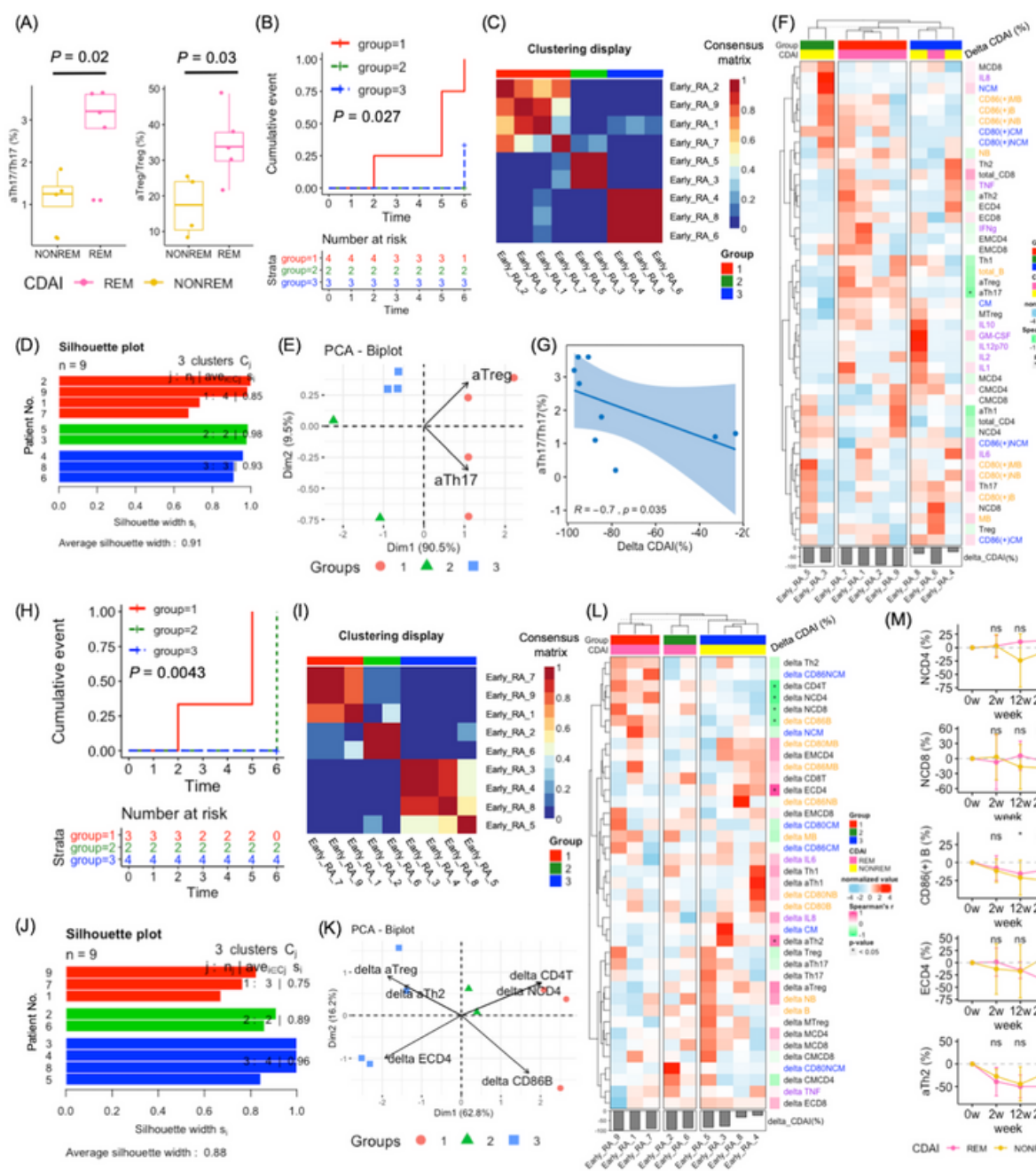

$\begin{array}{lll}\text { (L) } \sqrt{n+1} & \end{array}$

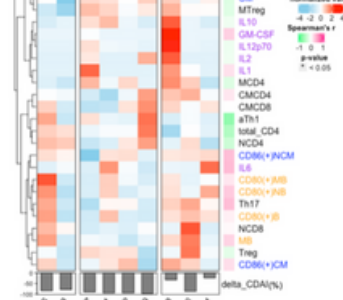

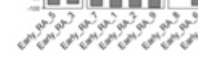
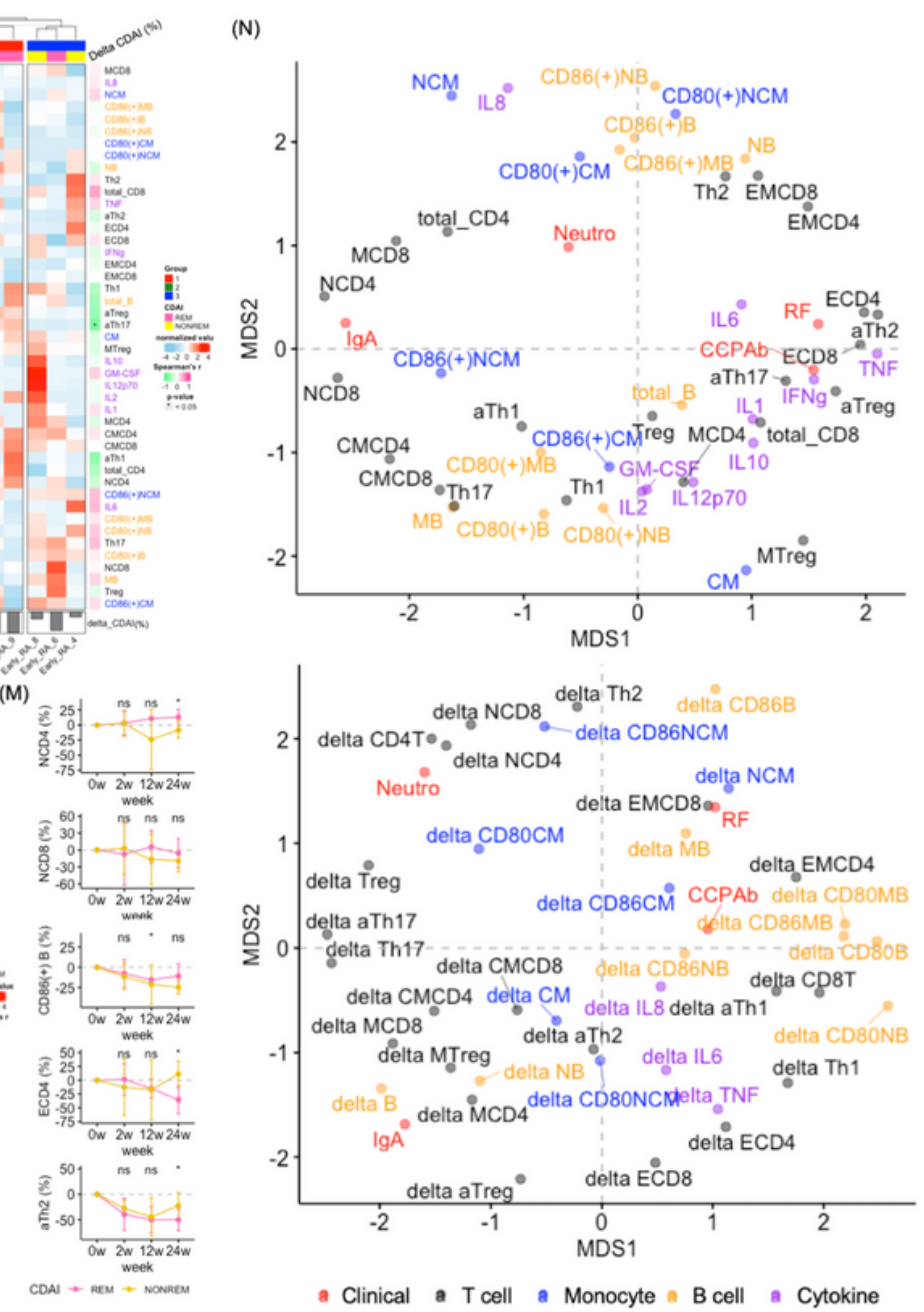

\section{Figure 3}

Peripheral immune-phenotyping and cytokines in patients with early RA. A) Comparison of the activated Th17 ratio and activated Treg ratio in early RA. P-value by the Student's t-test. B and H) Cumulative incidence plot for remission achievement according to subgroups of patients derived from pre-treatment dataset (B) and longitudinal dataset $(\mathrm{H})$. C and I) Heat-map of the patient similarity matrix derived from pre-treatment dataset (C) and longitudinal dataset (I). D and J) The Silhouette width shows whether a patient has been matched to its identified subgroup compared to other subgroups derived from pretreatment dataset (D) and longitudinal dataset $(\mathrm{J})$. A high value indicates that the patient has been wellmatched. E and K) the Principal component analysis by selected key features derived from pre-treatment dataset (E) and longitudinal dataset (K). In biplot, patients have been displayed as points while features 
have been displayed as vectors. $\mathrm{F}$ and $\mathrm{L}$ ) Hierarchical clustering analysis of pre-treatment dataset $(\mathrm{F})$ and longitudinal dataset $(\mathrm{L})$. Bottom boxplot represents the proportion of CDAl improvement 6 months after abatacept initiation. Heat-map on the right of main one represents how each parameter correlates with the proportion of CDAl improvement by depth of color; ' $'$ ': $p<0.05$ by the Spearman's correlation test. G) Scatter plot of the activated Th17 ratio and CDAl improvement of pre-treatment dataset. M) Longitudinal change of cell subpopulations. Results have been represented as median \pm interquartile range. The difference of their proportions between patients who achieved remission and others were compared by the Student's t-test: ' $*$ ': $p<0.05$. N) Visualization of multidimensional scaling demonstrating clustering of variables including laboratory tests, cell subpopulations and cytokines of pre-treatment dataset (above) and longitudinal dataset (below). Proximity between pairs of parameters was calculated using the Euclidean distances; the smaller proximity, the closer the distance between parameters. aTh1, activated Th1; aTh2, activated Th2; aTh17, activated Th17; aTreg, activated regulatory T cell; CCP, cyclic citrullinated peptide; CDAl, clinical disease activity score; $\mathrm{CM}$, classical monocyte; CMCD4, central memory CD4 T cell; CMCD8, central memory CD8 T cell; ECD4, effector CD4 T cell; ECD8, effector CD8 T cell; EMCD4, effector memory CD4 T cell; EMCD8, effector memory CD8 T cell; MB; memory B cell; MTreg, memory regulatory T cell; NB, naive B cell; NCD4, naive CD4 T cell; NCD8, naive CD8 T cell; NCM, nonclassical monocyte; NONREM, non-remission; RA, rheumatoid arthritis; REM, remission; Treg, regulatory $T$ cell. 

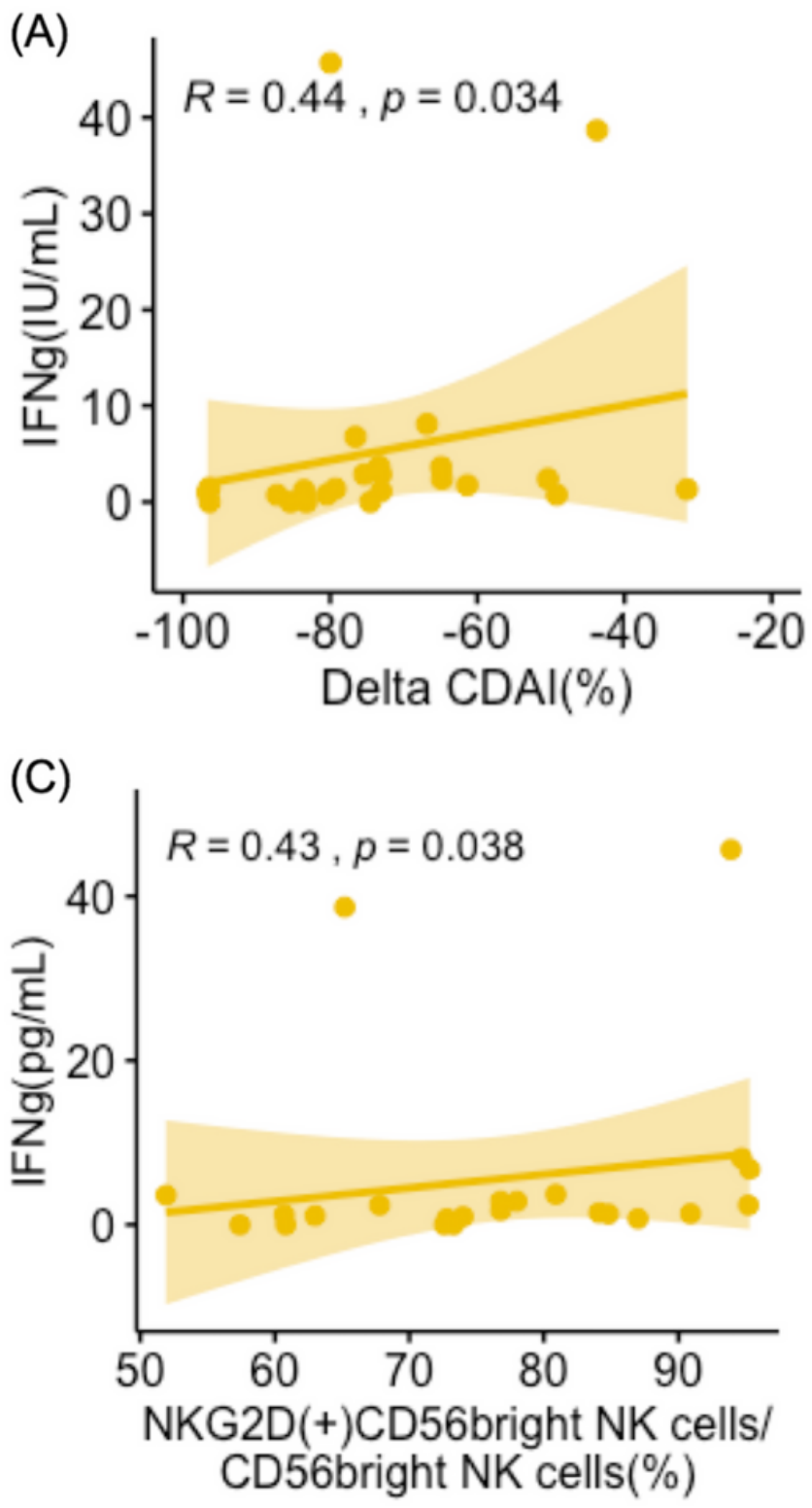

(B)
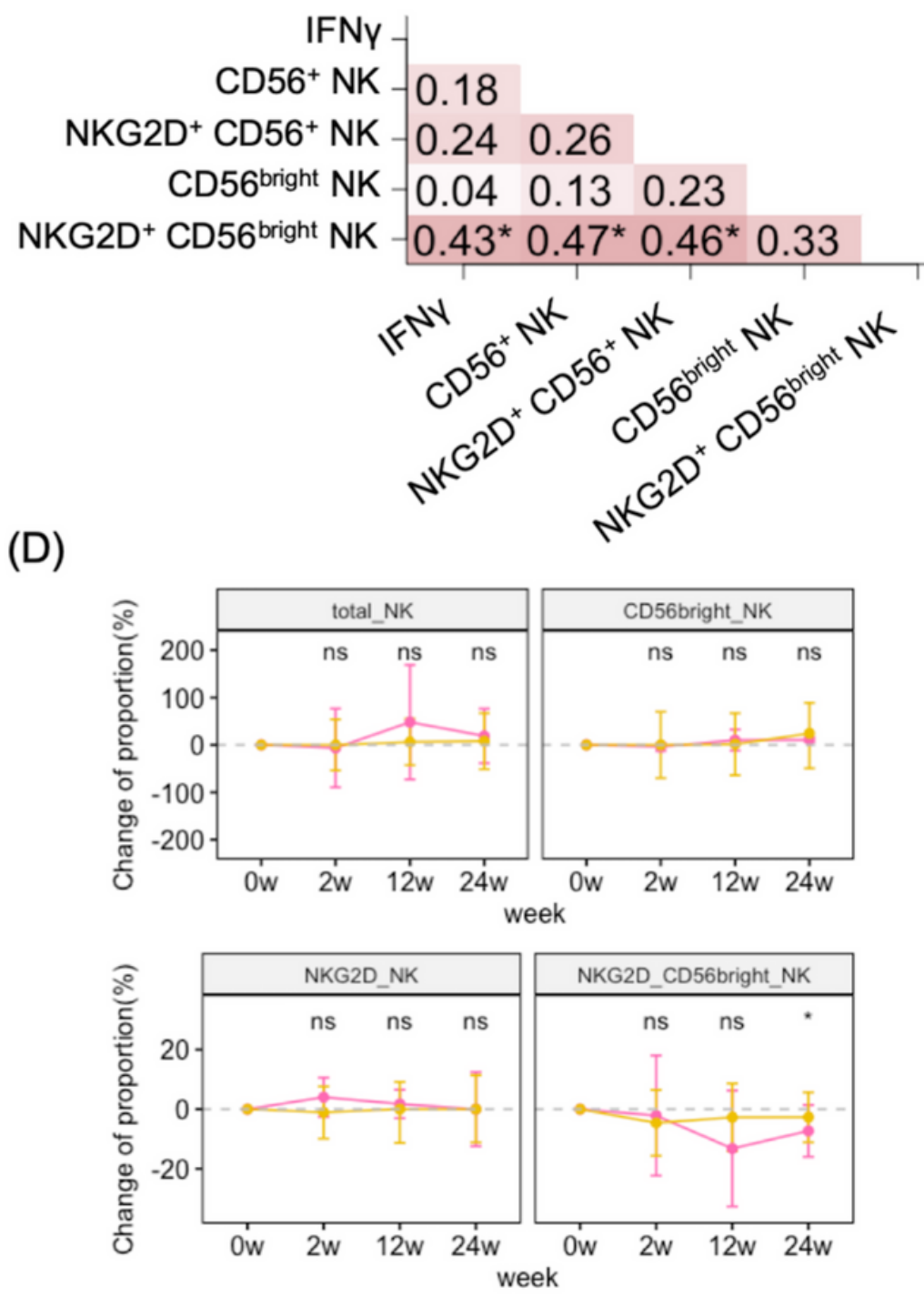

CDAI $\multimap$ REM $\bullet$ NONREM

\section{Figure 4}

Association among IFN- $y$ concentration and NK cell subpopulations in patients with established RA. A) Scatter plot of the concentration of IFN- $\gamma$ before treatment and the proportion of CDAl improvement. B) Correlation of IFN- $y$ concentrations and the proportion of CD56+ or CD56bright NK cell subpopulations. The number and color represent the Spearman's correlation coefficient; ' $* \star^{\prime}, p<0.01 ;{ }^{\prime}{ }^{\prime}, p<0.05$. C) Scatter plot of the concentration of IFN- $\gamma$ and the proportion of NKG2D+CD56bright NK cell subpopulations before treatment. D) Longitudinal change of NK cell subpopulations from baseline. Results have been represented as median \pm interquartile range. The difference of their proportions between patients who achieved remission and others was compared by the Student's t-test: ' $\star$ ': $p<0.05$. NK, natural killer cells; NKG2D, natural killer group 2, member $D$.

\section{Supplementary Files}


This is a list of supplementary files associated with this preprint. Click to download.

- SupplementaryTables.docx

- SuppleFig1.png

- SuppleFig2.png 